\title{
A FUNCTION FIELD ANALOGUE OF THE RASMUSSEN-TAMAGAWA CONJECTURE: THE DRINFELD MODULE CASE
}

\author{
Yoshiaki OKUMURA
}

(Received 20 May 2018 and revised 14 November 2018)

\begin{abstract}
In the arithmetic of function fields, Drinfeld modules play the role that elliptic curves play in the arithmetic of number fields. The aim of this paper is to study a nonexistence problem of Drinfeld modules with constraints on torsion points at places with large degree. This is motivated by a conjecture of Christopher Rasmussen and Akio Tamagawa on the non-existence of abelian varieties over number fields with some arithmetic constraints. We prove the non-existence of Drinfeld modules satisfying Rasmussen-Tamagawa type conditions in the case where the inseparable degree of the base field is not divisible by the rank of Drinfeld modules. Conversely if the rank divides the inseparable degree, then we prove the existence of a Drinfeld module satisfying Rasmussen-Tamagawa type conditions.
\end{abstract}

\section{Introduction}

Let $p$ be a prime number and fix some $p$-power $q=p^{m}$. Write $A:=\mathbb{F}_{q}[t]$ for the polynomial ring in one variable $t$ over $\mathbb{F}_{q}$ and set $F:=\mathbb{F}_{q}(t)$. Let $K$ be a finite extension of $F$. In this article, we identify every monic irreducible element $\pi$ of $A$ with the corresponding finite place of $F$. Write $\mathbb{F}_{\pi}=A / \pi A$ for the residue field at $\pi$ and set $q_{\pi}:=\# \mathbb{F}_{\pi}=q^{\operatorname{deg}(\pi)}$.

Let $r$ be a positive integer and $\pi$ a monic irreducible element of $A$. Define $\mathscr{D}(K, r, \pi)$ to be the set of isomorphism classes $[\phi]$ of rank- $r$ Drinfeld modules over $K$ which satisfy the following two conditions:

(D1) $\phi$ has good reduction at any finite place of $K$ not lying above $\pi$;

(D2) the $\bmod \pi$ representation $\bar{\rho}_{\phi, \pi}: G_{K} \rightarrow \mathrm{GL}_{r}\left(\mathbb{F}_{\pi}\right)$ attached to $\phi$ is of the form

$$
\bar{\rho}_{\phi, \pi} \simeq\left(\begin{array}{cccc}
\chi_{\pi}^{i_{1}} & * & \cdots & * \\
& \chi_{\pi}^{i_{2}} & \ddots & \vdots \\
& & \ddots & * \\
& & & \chi_{\pi}^{i_{r}}
\end{array}\right),
$$

where $\chi_{\pi}$ is the mod $\pi$ Carlitz character (see Example 2.7) and $0 \leq i_{1}, \ldots, i_{r} \leq$ $q_{\pi}-1$ are integers.

2010 Mathematics Subject Classification: Primary 11G09; Secondary 11R58.

Keywords: Drinfeld modules; Rasmussen-Tamagawa conjecture; Galois representations.

(C) 2019 Faculty of Mathematics, Kyushu University 
Consider the following question.

Question 1.1. Does there exist a positive constant $C>0$ depending only on $K$ and $r$ which satisfies the following: if $\operatorname{deg}(\pi)>C$, then the set $\mathscr{D}(K, r, \pi)$ is empty?

This question is motivated by a non-existence conjecture on abelian varieties stated by Rasmussen and Tamagawa [RT1]. Let $k$ be a finite extension of $\mathbb{Q}$ and $g$ a positive integer. For a prime number $\ell$, denote by $\tilde{k}_{\ell}$ the maximal pro- $\ell$ extension of $k\left(\mu_{\ell}\right)$ which is unramified outside $\ell$, where $\mu_{\ell}=\mu_{\ell}(\bar{k})$ is the set of $\ell$ th roots of unity in $\bar{k}$. For an abelian variety $X$ over $k$, write $k\left(X\left[\ell^{\infty}\right]\right):=k\left(\bigcup_{n \geq 1} X\left[\ell^{n}\right]\right)$ for the field generated by all $\ell$-power torsion points of $X$. Define $\mathscr{A}(k, g, \ell)$ to be the set of isomorphism classes [X] of $g$-dimensional abelian varieties over $k$ which satisfy the following equivalent conditions:

(RT-1) $k\left(X\left[\ell^{\infty}\right]\right) \subseteq \tilde{k}_{\ell}$;

(RT-2) $X$ has good reduction at any finite place of $k$ not lying above $\ell$ and $k(X[\ell]) / k\left(\mu_{\ell}\right)$ is an $\ell$-extension;

(RT-3) $X$ has good reduction at any finite place of $k$ not lying above $\ell$ and the mod $\ell$ representation $\bar{\rho}_{X, \ell}: G_{k} \rightarrow \mathrm{GL}_{\mathbb{F}_{\ell}}(X[\ell]) \simeq \mathrm{GL}_{2 g}\left(\mathbb{F}_{\ell}\right)$ is of the form

$$
\bar{\rho}_{X, \ell} \simeq\left(\begin{array}{cccc}
\chi_{\ell}^{i_{1}} & * & \cdots & * \\
& \chi_{\ell}^{i_{2}} & \ddots & \vdots \\
& & \ddots & * \\
& & & \chi_{\ell}^{i_{2 g}}
\end{array}\right),
$$

where $\chi_{\ell}$ is the $\bmod \ell$ cyclotomic character.

These conditions come from the study of a question of Ihara [Ih] related with the kernel of the canonical outer Galois representation of the pro- $\ell$ fundamental group of $\mathbb{P}^{1} \backslash\{0,1, \infty\}$; see [RT1]. Rasmussen and Tamagawa conjectured the following.

Conjecture 1.2. (Rasmussen and Tamagawa [RT1, Conjecture 1]) The set $\mathscr{A}(k, g, \ell)$ is empty for any $\ell$ large enough.

Since the set $\mathscr{A}(k, g, \ell)$ is always finite (see Section 5, or [RT1]), the conjecture is equivalent to saying that the union $\bigcup_{\ell} \mathscr{A}(k, g, \ell)$ is also finite. For example, the following cases are known:

- $\quad k=\mathbb{Q}$ and $g=1$ [RT1, Theorem 2];

- $\quad k=\mathbb{Q}$ and $g=2,3$ [RT2, Theorem 7.1 and Theorem 7.2];

- for abelian varieties with everywhere semistable reduction [Oz1, Corollary 4.5] and [RT2, Theorem 3.6];

- $\quad$ for abelian varieties with abelian Galois representations [Oz2, Corollary 1.3];

- for QM abelian surfaces over certain imaginary quadratic fields [Ar, Theorem 9.3].

We notice that, under the assumption of the generalized Riemann hypothesis (GRH) for Dedekind zeta functions of number fields, the conjecture is true in general [RT2, Theorem 5.1]. The key tool of this proof is the effective version of the Chebotarev density theorem for number fields, which holds under GRH. Rasmussen and Tamagawa also state the 'uniform version' of the conjecture [RT2, Conjecture 2], which says that one can take a lower bound of $\ell$ satisfying $\mathscr{A}(k, g, \ell)=\varnothing$ depending only on the degree $[k: \mathbb{Q}]$ and $g$. For instance, 
the uniform version of the conjecture for $\mathrm{CM}$ abelian varieties is proved by Bourdon [Bo, Corollary 1] and Lombardo [Lo, Theorem 1.3]. Under GRH, the uniform version of the conjecture is true if $[k: \mathbb{Q}]$ is odd [RT2, Theorem 5.2].

The arithmetic properties of Drinfeld modules are similar to those of elliptic curves over number fields. Under this analogy, the condition (D1)+(D2) can be regarded as a natural translation of the condition (RT-3). In fact, we have also Drinfeld module versions of (RT-1) and (RT-2); we will show that the set $\mathscr{D}(K, r, \pi)$ is characterized by three equivalent conditions as in the abelian variety case (Proposition 5.3).

The main purpose of this paper is to give a complete answer to Question 1.1.

THEOREM 1.3. (Theorem 3.7 and Theorem 4.9) If $r$ does not divide the inseparable degree $[K: F]_{\mathrm{i}}$ of $K / F$, then the set $\mathscr{D}(K, r, \pi)$ is empty for any $\pi$ whose degree is large enough.

THEOREM 1.4. (Theorem 5.6) If $r$ divides $[K: F]_{\mathrm{i}}$, then the set $\mathscr{D}(K, r, \pi)$ is never empty for any $\pi$.

The proof of Theorem 1.3 consists of the two cases: (i) $r=p^{v}$, and (ii) $r=r_{0} \cdot p^{v}$ for some $r_{0}>1$ which is prime to $p$. In the case (ii), we employ the strategy in [RT2] and use the effective version of the Chebotarev density theorem for function fields proved by Kumer and Scherk [KS]. In this case, the uniform version, which is an analogue of [RT2, Theorem 5.2], is also shown (Theorem 4.10). However, the same argument does not work well in the case (i). The proof in the case (i) is provided by observations about the tame inertia weights of $\bar{\rho}_{\phi, \pi}$ for any $[\phi] \in \mathscr{D}(K, r, \pi)$. This technique is used in [Oz1] and [RT2].

There are differences between the number field setting and the function field setting. Indeed, if $r$ divides $[K: F]_{\mathrm{i}}$ (for which there is no number field setting), then we construct a rank- $r$ Drinfeld module $\Phi$ over $K$ satisfying (D1) and (D2) for any $\pi$ and prove Theorem 1.4. This means that the Drinfeld module analogue of the Rasmussen-Tamagawa conjecture is not true in this case although the original conjecture is generally true under GRH.

The organization of the paper is as follows. In Section 2, after reviewing several basic facts on Drinfeld modules, we study the ramification of Galois representations coming from Drinfeld modules. The results are needed in the next section. In Section 3, for any $[\phi] \in \mathscr{D}(K, r, \pi)$, an important integer $e_{\phi}$ is introduced and we prove some non-trivial properties of it. This implies the result in the case (i). The aim of Section 4 is to give the proof in the case (ii). For any $[\phi] \in \mathscr{D}(K, r, \pi)$, we introduce a character $\chi\left(m_{\phi}\right)$ and show the property that $\chi\left(m_{\phi}\right)$ never vanishes on the Frobenius elements of places with some conditions. It contradicts a consequence of the effective Chebotarev density theorem if $\operatorname{deg}(\pi)$ is sufficiently large. Finally, in Section 5, we construct a Drinfeld module satisfying both (D1) and (D2) for any $\pi$ in the case where $r$ divides $[K: F]_{\mathrm{i}}$. We also show that the set $\mathscr{D}(K, r, \pi)$ is infinite if $\pi=t$ and $r \geq 2$.

\section{Notation}

Let $p, q, A, F$, and $K$ be as above. Set $n_{K}:=[K: F]$ and write $K_{\mathrm{S}}$ for the separable closure of $F$ in $K$. For a finite place $u$ of $K$ above $\pi$, let $K_{u}$ be the completion of $K$ at $u, \mathcal{O}_{K_{u}}$ its valuation ring, and $\mathbb{F}_{u}$ its residue field. We use the same symbol $u$ for the normalized valuation of $K_{u}$. Set $q_{u}:=\# \mathbb{F}_{u}$. Identify $G_{K_{u}}$ with the decomposition group of $G_{K}$ at $u$ and regard it 
as a subgroup of $G_{K}$. Denote by $I_{K_{u}}$ the inertia subgroup of $G_{K_{u}}$ at $u$ and choose a lift Frob $_{u} \in G_{K_{u}}$ of the Frobenius element of $G_{K_{u}} / I_{K_{u}}$. Denote by $e_{u \mid \pi}$ the absolute ramification index of $u$ and set $f_{u \mid \pi}:=\left[\mathbb{F}_{u}: \mathbb{F}_{\pi}\right]$.

Let $F_{\infty}:=\mathbb{F}_{q}((1 / t))$ be the completion of $F$ at the infinite place $\infty$ of $F$ and $\mathbb{C}_{\infty}$ the completion of a fixed algebraic closure of $F_{\infty}$. Every algebraic extension of $F$ is always regarded as a subfield of $\mathbb{C}_{\infty}$. Let $|\cdot|$ be the absolute value of $F_{\infty}$ attached to the normalized valuation of $F_{\infty}$. We also denote by $|\cdot|$ the unique extension of it to $\mathbb{C}_{\infty}$ and its restriction to each algebraic extension of $F$. For any non-zero $a \in A$, we see that $|a|=\#(A / a A)=q^{\operatorname{deg}(a)}$.

For any field $L$, denote by $G_{L}:=\operatorname{Gal}\left(L^{\mathrm{sep}} / L\right)$ the absolute Galois group of $L$. The notation $C=C(x, y, \ldots, z)$ indicates a constant $C$ depending only on $x, y, \ldots$, and $z$. We use the notation $\rho^{\mathrm{ss}}$ for the semisimplification of a representation $\rho$.

\section{Drinfeld modules}

\subsection{Basic definitions}

Let $L$ be a field equipped with an $\mathbb{F}_{q}$-algebra homomorphism $\iota: A \rightarrow L$. Such a pair $(L, \iota)$ is called an $A$-field. Let $\mathbb{G}_{a, L}$ be the additive group scheme defined over $L$. Denote by $\operatorname{End}_{\mathbb{F}_{q}}\left(\mathbb{G}_{a, L}\right)$ the ring of $\mathbb{F}_{q}$-linear endomorphisms of $\mathbb{G}_{a, L}$. It is isomorphic to the noncommutative polynomial ring $L\{\tau\}$ in one variable $\tau$ satisfying $\tau c=c^{q} \tau$ for any $c \in L$, where $\tau$ is the $q$-power Frobenius map. Let $r$ be a positive integer.

Definition 2.1. A Drinfeld module $\phi$ of rank $r$ defined over the $A$-field $L$ is an $\mathbb{F}_{q}$-algebra homomorphism

$$
\phi: A \rightarrow L\{\tau\} ; \quad a \mapsto \phi_{a}
$$

such that $\phi_{t}=\iota(t)+c_{1} \tau+\cdots+c_{r} \tau^{r}$, where $c_{1}, \ldots, c_{r} \in L$ and $c_{r} \neq 0$.

Note that $\phi$ is completely determined by the image $\phi_{t}$ of $t$. For two Drinfeld modules $\phi$ and $\psi$ over $L$, a morphism from $\phi$ to $\psi$ is an element $f \in L\{\tau\}$ such that $f \phi_{a}=\psi_{a} f$ for any $a \in A$. We say that $f$ is an isomorphism if there exists a morphism $g$ from $\psi$ to $\phi$ such that $f g=g f=1$. It is easy to check that $f$ is an isomorphism if and only if $f \in L^{\times}$.

For any $a \in A$, its image $\phi_{a}$ is an endomorphism of $\mathbb{G}_{a, L}$, so that $\phi$ endows the additive group $\mathbb{G}_{\mathrm{a}, L}\left(L^{\mathrm{sep}}\right)=L^{\text {sep }}$ with a new $A$-module structure defined by $a \cdot \lambda:=\phi_{a}(\lambda)$. Denote this $A$-module by ${ }_{\phi} L^{\text {sep }}$. For any non-zero element $a \in A$, the set of $a$-torsion points

$$
\phi[a]=\left\{\lambda \in{ }_{\phi} L^{\mathrm{sep}} ; a \cdot \lambda=\phi_{a}(\lambda)=0\right\}
$$

of $\phi$ is an $A$-submodule of ${ }_{\phi} L^{\text {sep }}$ on which $G_{L}$ acts. If $a$ is not contained in the kernel of $\iota$, then $\phi[a]$ is a free $A / a A$-module of rank $r$.

Let $K$ be a finite extension of $F$. From now on, unless otherwise stated, we regard $K$ as an $A$-field via the inclusion $A \hookrightarrow F \subset K$. Let $\phi$ be a rank- $r$ Drinfeld module over $K$. For any finite place $v$ of $K$, we can regard $\phi$ as a Drinfeld module over $K_{v}$ via the canonical inclusion $K\{\tau\} \hookrightarrow K_{v}\{\tau\}$.

Definition 2.2. (1) We say that $\phi$ has stable reduction at $v$ if there exists a Drinfeld module $\psi$ over $K_{v}$ such that $\psi$ is isomorphic to $\phi$ over $K_{v}$ and

$$
\psi_{t}=t+c_{1}^{\prime} \tau+\cdots+c_{r}^{\prime} \tau^{r}
$$


such that $c_{1}^{\prime}, \ldots, c_{r}^{\prime} \in \mathcal{O}_{K_{v}}$ and $c_{r^{\prime}}^{\prime} \in \mathcal{O}_{K_{v}}^{\times}$for some $1 \leq r^{\prime} \leq r$.

(2) We say that $\phi$ has good reduction at $v$ if it has stable reduction at $v$ and $c_{r}^{\prime} \in \mathcal{O}_{K_{v}}^{\times}$.

Proposition 2.3. (Drinfeld [Dr, Proposition 7.1]) Every Drinfeld module $\phi$ over $K$ has potentially stable reduction at any finite place $v$ of $K$.

Remark 2.4. Write $\phi_{t}=t+c_{1} \tau+\cdots+c_{r} \tau^{r}$ and set $R:=\min _{1 \leq s \leq r}\left\{v\left(c_{s}\right) /\left(q^{s}-1\right)\right\}$. Let $K_{v}^{\prime}$ be a finite extension of $K_{v}$. If the ramification index $e\left(K_{v}^{\prime} / K_{v}\right)$ satisfies $e\left(K_{v}^{\prime} / K_{v}\right) \cdot R \in \mathbb{Z}$, then $\phi$ has stable reduction over $K_{v}^{\prime}$. In particular, we can take $K_{v}^{\prime} / K_{v}$ as a tamely ramified finite separable extension whose ramification index $e\left(K_{v}^{\prime} / K_{v}\right)$ divides $\prod_{s=1}^{r}\left(q^{s}-1\right)$. Every rank-one Drinfeld module clearly has potentially good reduction at any finite place.

For any monic irreducible element $\pi \in A$, the set of $\pi$-torsion points $\phi[\pi]$ is a $G_{K}$-stable $r$-dimensional $\mathbb{F}_{\pi}$-vector space, so that the $\bmod \pi$ representation

$$
\bar{\rho}_{\phi, \pi}: G_{K} \rightarrow \operatorname{GL}_{\mathbb{F}_{\pi}}(\phi[\pi]) \simeq \mathrm{GL}_{r}\left(\mathbb{F}_{\pi}\right)
$$

attached to $\phi$ can be defined. Let $A_{\pi}:=\lim A / \pi^{n} A$ be the $\pi$-adic completion of $A$. Considering the maps $\phi\left[\pi^{n+1}\right] \rightarrow \phi\left[\pi^{n}\right]$ defined by $x \mapsto \pi \cdot x$, one can define the $\pi$-adic Tate module $T_{\pi}(\phi):=\lim \phi\left[\pi^{n}\right]$. It is a free $A_{\pi}$-module of rank $r$ with continuous $G_{K^{-}}$ action. Write $\rho_{\phi, \pi}: G_{K} \leftrightarrows \mathrm{GL}_{r}\left(A_{\pi}\right)$ for the representation attached to $T_{\pi}(\phi)$.

Let $\pi_{0}$ be a monic irreducible element of $A$ with $\pi_{0} \neq \pi$ and let $v$ be a finite place of $K$ above $\pi_{0}$. The next proposition is an analogue of the Néron-Ogg-Shafarevich criterion for good reduction of abelian varieties (cf. [ST, Theorem 1]).

Proposition 2.5. (Takahashi [Ta, Theorem 1]) A Drinfeld module $\phi$ over $K$ has good reduction at $v$ if and only if $T_{\pi}(\phi)$ is unramified at $v$.

Suppose that $\phi$ has good reduction at $v$. Then $\rho_{\phi, \pi}$ is unramified at $v$, and so $\rho_{\phi, \pi}\left(\right.$ Frob $\left._{v}\right) \in \rho_{\phi, \pi}\left(G_{K}\right)$ is independent of the choice of Frob ${ }_{v}$. Denote by

$$
P_{v}(T):=\operatorname{det}\left(T-\rho_{\phi, \pi}\left(\operatorname{Frob}_{v}\right) \mid T_{\pi}(\phi)\right) \in A_{\pi}[T]
$$

the characteristic polynomial of Frob ${ }_{v}$. Then we have the following proposition.

Proposition 2.6. (Takahashi [Ta, Proposition 3(ii)]) The polynomial $P_{v}(T)$ has coeffcients in $A$ and is independent of $\pi$. Any root $\alpha$ of $P_{v}(T)$ satisfies $|\alpha|=q_{v}^{1 / r}$.

The following example gives a function field analogue of cyclotomic extensions of number fields.

Example 2.7. (Cf. [Ro, Ch. 12]) The rank-one Drinfeld module $\mathcal{C}: A \rightarrow F\{\tau\}$ determined by $\mathcal{C}_{t}=t+\tau$ is called the Carlitz module. For any monic irreducible element $\pi \in A$, define

$$
\chi_{\pi}: G_{F} \rightarrow \mathrm{GL}_{\mathbb{F}_{\pi}}(\mathcal{C}[\pi]) \simeq \mathbb{F}_{\pi}^{\times} .
$$

It is called the $\bmod \pi$ Carlitz character. Since $\mathcal{C}$ has good reduction at any finite place $\pi_{0}$ of $F$, the character $\chi_{\pi}$ is unramified at $\pi_{0}$ if $\pi_{0} \neq \pi$. For any finite place $v$ of $K$ above $\pi_{0} \neq \pi$, it is known that $\chi_{\pi}$ satisfies

$$
\chi_{\pi}\left(\operatorname{Frob}_{v}\right) \equiv \pi_{0}^{f_{v \mid \pi_{0}}} \quad(\bmod \pi) .
$$


The mod $\pi$ Carlitz character induces an isomorphism $\operatorname{Gal}(F(\mathcal{C}[\pi]) / F) \stackrel{\sim}{\rightarrow} \mathbb{F}_{\pi}^{\times}$, so that $F(\mathcal{C}[\pi]) / F$ is a cyclic extension which is unramified outside $\pi$ and $\infty$. Moreover, it is known that $\pi$ is totally ramified in $F(\mathcal{C}[\pi])$ and the ramification of the infinite place $\infty$ is as follows: there exists a subfield $F(\mathcal{C}[\pi])_{+}$with degree $\left[F(\mathcal{C}[\pi]): F(\mathcal{C}[\pi])_{+}\right]=q-1$ such that $\infty$ is totally split in $F(\mathcal{C}[\pi])_{+}$and any place of $F(\mathcal{C}[\pi])_{+}$above $\infty$ is totally ramified in $F(\mathcal{C}[\pi])$.

\subsection{Tate uniformization}

Let $u$ be a finite place of $K$ above $\pi$ and $\phi$ a rank- $r$ Drinfeld module over $K$. Suppose that $\phi$ has stable reduction at $u$. Then Drinfeld's result on Tate uniformization gives an analytic description of $\phi$ as a Drinfeld module over $K_{u}$.

Proposition 2.8. (Tate uniformization; Drinfeld [Dr, Section 7]) There exist a unique Drinfeld module $\psi$ over $K_{u}$ with good reduction and a unique entire analytic surjective morphism $e: \psi \rightarrow \phi$ defined over $K_{u}$ such that e is the identity on $\operatorname{Lie}\left(\mathbb{G}_{a, K_{u}}\right)$.

It is known that the rank $r^{\prime}$ of $\psi$ satisfies $r^{\prime} \leq r$ and the kernel $H:=\operatorname{Ker}(e)\left(K_{u}^{\mathrm{sep}}\right)$ is an $A$-lattice of rank $h:=r-r^{\prime}$ in $\psi K_{u}^{\text {sep }}$, endowed with an action of a finite quotient of $G_{K_{u}}$. For any monic irreducible element $\pi_{0} \in A$, the analytic morphism $e$ induces the short exact sequence

$$
0 \rightarrow T_{\pi_{0}}(\psi) \rightarrow T_{\pi_{0}}(\phi) \rightarrow H \otimes_{A} A_{\pi_{0}} \rightarrow 0
$$

of $A_{\pi_{0}}\left[G_{K_{u}}\right]$-modules. In the case where $\pi_{0} \neq \pi$, the $I_{K_{u}}$-action on $T_{\pi_{0}}(\phi)$ is potentially unipotent since both $T_{\pi_{0}}(\psi)$ and $H \otimes_{A} A_{\pi_{0}}$ are potentially unramified at $u$.

Remark 2.9. By the theory of 'analytic $\tau$-sheaves' (see [Ga1], [Ga2] and [Ga3]), the sequence (2.1) can be interpreted as follows. For any Drinfeld module $\phi$ over $K_{u}$, one can construct an analytic $\tau$-sheaf $\tilde{M}(\phi)$ associated with $\phi$. It is a locally free $\mathscr{O}_{\tilde{\mathbb{A}}_{K_{u}}^{1}}$-module of finite rank on $\tilde{\mathbb{A}}_{K_{u}}^{1}$ with some additional structures, where $\tilde{\mathbb{A}}_{K_{u}}^{1}$ is the rigid analytic space associated with the affine line $\mathbb{A}_{K_{u}}^{1}=\operatorname{Spec} A \times \operatorname{Spec} \mathbb{F}_{q} \operatorname{Spec} K_{u}$. Then the $\pi_{0}$-adic Tate module $T_{\pi_{0}}(\tilde{M}(\phi))$ of $\tilde{M}(\phi)$ can be defined and it is canonically isomorphic to $T_{\pi_{0}}(\phi)$. The Tate uniformization implies that there exist an analytic $\tau$-sheaf $\tilde{N}$ which is potentially trivial and an exact sequence

$$
0 \rightarrow \tilde{N} \rightarrow \tilde{M}(\phi) \rightarrow \tilde{M}(\psi) \rightarrow 0
$$

Since $\tilde{M} \mapsto T_{\pi_{0}}(\tilde{M})$ is a contravariant exact functor, we obtain

$$
0 \rightarrow T_{\pi_{0}}(\tilde{M}(\psi)) \rightarrow T_{\pi_{0}}(\tilde{M}(\phi)) \rightarrow T_{\pi_{0}}(\tilde{N}) \rightarrow 0,
$$

which coincides with the sequence (2.1) (for example, see [Ga4, Example 7.1]).

We would like to estimate the tame ramification of the lattice $H$. Suppose that $H \neq 0$ and consider the representation

$$
\rho: I_{K_{u}} \rightarrow \mathrm{GL}_{A}(H) \simeq \mathrm{GL}_{h}(A) .
$$

Then we have the following proposition. 
Proposition 2.10. There exists a finite separable extension $L / K_{u}$ such that:

(1) the action of $I_{L}$ on $H$ is trivial;

(2) the ramification index $e\left(L_{0} / K_{u}\right)$ divides $\prod_{s=1}^{r-1}\left(q^{s}-1\right)$, where $L_{0}$ is the maximal tamely ramified extension of $K_{u}$ in $L$.

Proof. Let $E / K_{u}$ be a finite Galois extension such that the action of $G_{K_{u}}$ on $H$ factors through $\operatorname{Gal}\left(E / K_{u}\right)$. Now the image $I$ of $I_{K_{u}}$ by the canonical projection map $G_{K_{u}} \rightarrow$ $\operatorname{Gal}\left(E / K_{u}\right)$ is the inertia subgroup of $\operatorname{Gal}\left(E / K_{u}\right)$. For the representation

$$
\rho: I \rightarrow \mathrm{GL}_{A}(H) \simeq \mathrm{GL}_{h}(A),
$$

denote by $L$ the fixed subfield of $E$ by $J:=\operatorname{Ker}(\rho)$. By construction, the action of the inertia subgroup $I_{L}$ of $G_{L}$ on $H$ is trivial. Now the image $\operatorname{Im}(\rho)$ is a finite subgroup of $\mathrm{GL}_{h}(A)$ of order \#I/J=e(L/Ku) and $h \leq r-1$. Applying Lemma 2.12 below to $\operatorname{Im}(\rho)$, we see that $e\left(L_{0} / K_{u}\right)$ divides $\prod_{s=1}^{r-1}\left(q^{s}-1\right)$.

Remark 2.11. Proposition 2.10 means that the Drinfeld module $\phi$ is 'semistable' over $L$ in the following sense. By Remark 2.9, the analytic $\tau$-sheaf $\tilde{M}(\phi)$ is the extension of $\tilde{M}(\psi)$ by $\tilde{N}$ and both $\tilde{M}(\psi)$ and $\tilde{N}$ are 'good' over $L$. Hence the analytic $\tau$-sheaf $\tilde{M}(\phi)$ is strongly semistable over $L$ in the sense of [Ga3, Definition 4.6].

LEMMA 2.12. Let $n$ be a positive integer and let $G$ be a finite subgroup of $\operatorname{GL}_{n}(A)$. Then the maximal prime-to- $p$ divisor of $\# G$ is a factor of $\prod_{s=1}^{n}\left(q^{s}-1\right)$.

Proof. Consider the $t$-adic completion $\mathbb{F}_{q}[[t]]$ of $A$ and regard $G$ as a finite subgroup of $\mathrm{GL}_{n}\left(\mathbb{F}_{q}[[t]]\right)$. Let $\Gamma_{n}$ be the kernel of the map $\mathrm{GL}_{n}\left(\mathbb{F}_{q}[[t]]\right) \rightarrow \mathrm{GL}_{n}\left(\mathbb{F}_{q}\right)$ induced by the reduction map $\mathbb{F}_{q}[[t]] \rightarrow \mathbb{F}_{q}$. Since $\mathbb{F}_{q}[[t]]$ is a complete noetherian local ring whose residue field is finite of characteristic $p, \Gamma_{n}$ is a pro- $p$ group. Hence the short exact sequence $1 \rightarrow \Gamma_{n} \rightarrow \mathrm{GL}_{n}\left(\mathbb{F}_{q}[[t]]\right) \rightarrow \mathrm{GL}_{n}\left(\mathbb{F}_{q}\right) \rightarrow 1$ shows that the maximal prime-to- $p$ divisor of $\# G$ is a factor of $\mathrm{ZL}_{n}\left(\mathbb{F}_{q}\right)=q^{n(n-1) / 2} \prod_{s=1}^{n}\left(q^{s}-1\right)$.

\section{Inertia action on torsion points}

Let $\pi$ be a monic irreducible element of $A$. In this section, studying the ramification of mod $\pi$ representations attached to Drinfeld modules, we show the non-existence result (Theorem 3.7) in the case where $r$ is a $p$-power and does not divide $[K: F]_{i}$.

\subsection{Tame inertia weights}

Let $u$ be a finite place of $K$ above $\pi$. For a fixed separable closure $K_{u}^{\text {sep }}$ of $K_{u}$ with residue field $\overline{\mathbb{F}}_{u}$, denote by $K_{u}^{\text {ur }}$ (respectively $K_{u}^{\mathrm{t}}$ ) the maximal unramified (respectively maximal tamely ramified) extension of $K_{u}$ in $K_{u}^{\text {sep }}$, so that $I_{K_{u}}$ is isomorphic to $\operatorname{Gal}\left(K_{u}^{\text {sep }} / K_{u}^{\text {ur }}\right)$. Denote by $I^{\mathrm{t}}:=\mathrm{Gal}\left(K_{u}^{\mathrm{t}} / K_{u}^{\mathrm{ur}}\right)$ the tame inertia subgroup of $I_{K_{u}}$. Let $d$ be a positive integer and $\mathbb{F}$ the finite field with $q_{\pi}^{d}$ elements in $\overline{\mathbb{F}}_{u}$. Then $\mathbb{F}$ is the finite extension of $\mathbb{F}_{\pi}$ of degree $d$. Write $\mu_{q_{\pi}^{d}-1}\left(K_{u}^{\text {sep }}\right)$ for the set of $\left(q_{\pi}^{d}-1\right)$ th roots of unity in $K_{u}^{\text {sep }}$ and fix the isomorphism $\mu_{q_{\pi}^{d}-1}\left(K_{u}^{\text {sep }}\right) \stackrel{\sim}{\rightarrow} \mathbb{F}^{\times}$coming from the reduction map $\mathcal{O}_{K_{u}^{\text {sep }}} \rightarrow \overline{\mathbb{F}}_{u}$. For a uniformizer $\varpi$ of 
$K_{u}$, choose a solution $\eta \in K_{u}^{\text {sep }}$ to the equation $X^{q_{\pi}^{d}-1}-\varpi=0$ and define

$$
\omega_{d, K_{u}}: I_{K_{u}} \rightarrow \mu_{q_{\pi}^{d}-1}\left(K_{u}^{\mathrm{sep}}\right) \stackrel{\sim}{\rightarrow} \mathbb{F}^{\times} ; \quad \sigma \mapsto \frac{\eta^{\sigma}}{\eta} .
$$

It is independent of the choices of $\varpi$ and $\eta$. The character $\omega_{d, K_{u}}$ factors through $I^{\mathrm{t}}$ (cf. [Se1]). We call the $\operatorname{Gal}\left(\mathbb{F} / \mathbb{F}_{\pi}\right)$-conjugates $\left(\omega_{d, K_{u}}\right)^{q_{\pi}^{i}}$ for $0 \leq i \leq d-1$ of $\omega_{d, K_{u}}$ the fundamental characters of level $d$. It is easy to check that

$$
\left(\omega_{d, K_{u}}\right)^{1+q_{\pi}+\cdots+q_{\pi}^{d-1}}=\omega_{1, K_{u}}
$$

and $\left(\omega_{d, K_{u}}\right)^{q_{\pi}^{d}-1}=1$. For any finite extension $L$ of $K_{u}$, we see that $\left.\left(\omega_{d, K_{u}}\right)\right|_{I_{L}}=$ $\left(\omega_{d, L}\right)^{e\left(L / K_{u}\right)}$ by definition.

As an analogue of Serre's classical result on the $\bmod \ell$ cyclotomic character [Se1, Proposition 8], the following fact is known.

Proposition 3.1. (Kim [Ki, Proposition 9.4.3.(2)]) The character $\left(\omega_{1, K_{u}}\right)^{e_{u \mid \pi}}$ coincides with the mod $\pi$ Lubin-Tate character restricted to $I_{K_{u}}$.

Remark 3.2. The mod $\pi$ Lubin-Tate character is the character describing the $G_{K_{u}}$ action on the $\pi$-torsion points of the Lubin-Tate formal group over $\mathcal{O}_{K_{u}}$ associated with $\pi$. It coincides with the mod $\pi$ Carlitz character $\chi_{\pi}$ restricted to $G_{K_{u}}$, so that $\chi_{\pi}=\left(\omega_{1, K_{u}}\right)^{e_{u \mid \pi}}$ on $I_{K_{u}}$.

Let $V$ be a $d$-dimensional irreducible $\mathbb{F}_{\pi}$-representation of $I_{K_{u}}$. Then the action of $I_{K_{u}}$ on $V$ factors through $I^{\mathrm{t}}$, so that $V$ can be regarded as a representation of $I^{\mathrm{t}}$. Using Schur's lemma, we see that $\operatorname{End}_{I^{\mathrm{t}}}(V)$ is a finite field of order $q_{\pi}^{d}$. Fix an isomorphism $f: \operatorname{End}_{I^{t}}(V) \stackrel{\sim}{\rightarrow} \mathbb{F}$ and regard $V$ as a one-dimensional $\mathbb{F}$-representation

$$
\rho: I^{\mathrm{t}} \rightarrow \operatorname{End}_{I^{\mathrm{t}}}(V)^{\times} \stackrel{\sim}{\rightarrow} \mathbb{F}^{\times}
$$

of $I^{\mathrm{t}}$. Since $I^{\mathrm{t}}$ is pro-cyclic and $\omega_{d, K_{u}}$ is surjective, there exists an integer $0 \leq j \leq q_{\pi}^{d}-2$ such that $\rho=\left(\omega_{d, K_{u}}\right)^{j}$. If we decompose $j=n_{0}+n_{1} q_{\pi}+\cdots+n_{d-1} q_{\pi}^{d-1}$ with integers $0 \leq n_{s} \leq q_{\pi}-1$, then the set $\left\{n_{0}, n_{1}, \ldots, n_{d-1}\right\}$ is independent of the choice of $f$.

Definition 3.3. For a $d$-dimensional irreducible $\mathbb{F}_{\pi}$-representation $V$ of $I_{K_{u}}$, the integers $n_{0}, n_{1}, \ldots, n_{d-1}$ as above are called the tame inertia weights of $V$. For any $\mathbb{F}_{\pi^{-}}$ representation $\rho: G_{K_{u}} \rightarrow \mathrm{GL}_{\mathbb{F}_{\pi}}(V)$, the tame inertia weights of $\rho$ are the tame inertia weights of all the Jordan-Hölder quotients of $\left.V\right|_{I_{K_{u}}}$.

Denote by $\mathrm{TI}_{K_{u}}(\rho)$ the set of tame inertia weights of $\rho: G_{K_{u}} \rightarrow \mathrm{GL}_{d}\left(\mathbb{F}_{\pi}\right)$.

\subsection{Ramification of constrained torsion points}

Let $\phi$ be a Drinfeld module over $K$ satisfying $[\phi] \in \mathscr{D}(K, r, \pi)$ and $u$ a finite place of $K$ above $\pi$. By Remark 2.4, we can take a finite separable extension $K_{u}^{\prime}$ of $K_{u}$ such that $\phi$ has stable reduction and $e\left(K_{u}^{\prime} / K_{u}\right)$ divides $\prod_{s=1}^{r}\left(q^{s}-1\right)$. By Tate uniformization, we obtain the exact sequence

$$
0 \rightarrow \psi[\pi] \rightarrow \phi[\pi] \rightarrow H \otimes_{A} \mathbb{F}_{\pi} \rightarrow 0
$$


of $\mathbb{F}_{\pi}\left[G_{K_{u}^{\prime}}\right]$-modules. We also take a finite separable extension $L$ of $K_{u}^{\prime}$ as in Proposition 2.10 and denote by $L_{0}$ the maximal tamely ramified extension of $K_{u}^{\prime}$ in $L$. Set

$$
C_{1}=C_{1}(q, r):=\left(q^{r}-1\right) \prod_{s=1}^{r-1}\left(q^{s}-1\right)^{2}
$$

and $e_{u}:=e\left(L_{0} / K_{u}^{\prime}\right) \cdot e\left(K_{u}^{\prime} / F_{\pi}\right)$. Then $e_{u}$ divides $e_{u \mid \pi} C_{1}(q, r)$.

Proposition 3.4. Every tame inertia weight of $\left.\bar{\rho}_{\phi, \pi}\right|_{G_{L_{0}}}$ is between 0 and $e_{u}$.

Proof. By (D2), the restriction $\left.\bar{\rho}_{\phi, \pi}^{\mathrm{ss}}\right|_{I_{L_{0}}}$ is isomorphic to $\left(\omega_{1, L_{0}}\right)^{j_{1}} \oplus \cdots \oplus\left(\omega_{1, L_{0}}\right)^{j_{r}}$, where $\left\{j_{1}, \ldots, j_{r}\right\}=\mathrm{TI}_{L_{0}}\left(\bar{\rho}_{\phi, \pi}\right)$. Write $\bar{\rho}: G_{L_{0}} \rightarrow \mathrm{GL}_{h}\left(\mathbb{F}_{\pi}\right)$ for the representation arising from $H \otimes_{A} \mathbb{F}_{\pi}$. Then the sequence (3.1) implies $\bar{\rho}_{\phi, \pi}^{\mathrm{ss}}=\bar{\rho}_{\psi, \pi}^{\mathrm{ss}} \oplus \bar{\rho}^{\mathrm{ss}}$ on $G_{L_{0}}$, so that $\operatorname{TI}_{L_{0}}\left(\bar{\rho}_{\phi, \pi}\right)=$ $\mathrm{TI}_{L_{0}}\left(\bar{\rho}_{\psi, \pi}\right) \cup \mathrm{TI}_{L_{0}}(\bar{\rho})$. Let $\tilde{M}(\psi)$ and $\tilde{N}$ be analytic $\tau$-sheaves on $\tilde{\mathbb{A}}_{L_{0}}^{1}$ attached to $\psi$ and $H$, respectively. Since $\tilde{M}(\psi)$ is good over $L_{0}$, we see that $\mathrm{TI}_{L_{0}}\left(\bar{\rho}_{\psi, \pi}\right) \subset\left[0, e_{u}\right]$ by [Ga1, Theorem 2.14]. On the other hand, the analytic $\tau$-sheaf $\tilde{N}$ is of dimension zero and good over $L$, so that every tame inertia weight of $\left.\bar{\rho}\right|_{G_{L}}$ is zero by [Ga1, Theorem 2.14]. This means that $\left.\bar{\rho}^{\mathrm{ss}}\right|_{I_{L}}=1$. Hence we see that

$$
\left.\left(\omega_{1, L_{0}}\right)^{j}\right|_{I_{L}}=\left(\omega_{1, L}\right)^{e\left(L / L_{0}\right) \cdot j}=1
$$

for any $j \in \mathrm{TI}_{L_{0}}(\bar{\rho})$, so that $e\left(L / L_{0}\right) \cdot j \equiv 0\left(\bmod q_{\pi}-1\right)$ holds. Since $e\left(L / L_{0}\right)$ is a $p$ power and $0 \leq j \leq q_{\pi}-2$, we see that $j=0$.

The condition (D2) means that $\bar{\rho}_{\phi, \pi}^{\mathrm{ss}}$ is isomorphic to $\chi_{\pi}^{i_{1}} \oplus \cdots \oplus \chi_{\pi}^{i_{r}}$ for $0 \leq$ $i_{1}, \ldots, i_{r} \leq q_{\pi}-1$. By renumbering $\left\{j_{1}, \ldots, j_{r}\right\}$ if necessary, Propositions 3.1 and 3.4 mean that $\left.\chi_{\pi}^{i_{s}}\right|_{L_{0}}=\left(\omega_{1, L_{0}}\right)^{i_{s} \cdot e_{u}}=\left(\omega_{1, L_{0}}\right)^{j_{s}}$ for any $1 \leq s \leq r$. Thus we obtain

$$
i_{s} \cdot e_{u} \equiv j_{s} \quad\left(\bmod q_{\pi}-1\right)
$$

for any $1 \leq s \leq r$.

For any finite place $v$ of $K$ not lying above $\pi$ and any integer $m$, denote by $P_{v, m}(T)=$ $\operatorname{det}\left(T-\rho_{\phi, \pi}\left(\operatorname{Frob}_{v}^{m}\right) \mid T_{\pi}(\phi)\right) \in A[T]$ the characteristic polynomial of $\operatorname{Frob}_{v}^{m}$. Set

$$
C_{2}=C_{2}\left(n_{K}, q, r\right):=r \cdot n_{K}^{2} \cdot C_{1}(q, r) .
$$

Then we obtain the following important proposition.

PROPOSITION 3.5. If $\operatorname{deg}(\pi)>C_{2}$, then $r$ divides $e_{u}$ and the congruence

$$
i_{s} \cdot e_{u} \equiv \frac{e_{u}}{r} \quad\left(\bmod q_{\pi}-1\right)
$$

holds for any $1 \leq s \leq r$.

Proof. Suppose that $\operatorname{deg}(\pi)>C_{2}$. Take a monic irreducible element $\pi_{0} \in A$ with $\operatorname{deg}\left(\pi_{0}\right)$ $=1$ and a finite place $v$ of $K$ above $\pi_{0}$. Since $\phi$ has good reduction at $v$ by (D1), the polynomial $P_{v, e_{u}}(T)$ is well defined. Now the roots of $P_{v, e_{u}}(T)$ are given by $\left\{\alpha_{s}^{e_{u}}\right\}_{s=1}^{r}$, where $\left\{\alpha_{s}\right\}_{s=1}^{r}$ are the roots of $P_{v}(T)=P_{v, 1}(T)$. On the other hand, the condition (D2) implies that the roots of the polynomial $P_{v, e_{u}}(T)(\bmod \pi)$ in $\mathbb{F}_{\pi}[T]$ are given by $\left\{\chi_{\pi}\left(\operatorname{Frob}_{v}\right)^{i_{s} \cdot e_{u}}\right\}_{s=1}^{r}$. Set $\pi_{v}:=\pi_{0}^{f_{v \mid \pi_{0}}}$. By the above relation (3.2), we see that $\chi_{\pi}\left(\operatorname{Frob}_{v}\right)^{i_{s} \cdot e_{u}}=\chi_{\pi}\left(\operatorname{Frob}_{v}\right)^{j_{s}}$ for 
any $1 \leq s \leq r$. Since $\chi_{\pi}\left(\text { Frob }_{v}\right)^{j_{s}} \equiv \pi_{v}^{j_{s}}(\bmod \pi)$ holds by Example 2.7, we obtain

$$
P_{v, e_{u}}(T) \equiv \prod_{s=1}^{r}\left(T-\chi_{\pi}\left(\operatorname{Frob}_{v}\right)^{j_{s}}\right) \equiv \prod_{s=1}^{r}\left(T-\pi_{v}^{j_{s}}\right) \quad(\bmod \pi) .
$$

Denote by $S_{k}\left(x_{1}, \ldots, x_{r}\right)$ the fundamental symmetric polynomial of degree $k$ with $r$ variables $x_{1}, \ldots, x_{r}$ for $0 \leq k \leq r$. Then

$$
\prod_{s=1}^{r}\left(T-x_{s}\right)=\sum_{k=0}^{r}(-1)^{k} S_{k}\left(x_{1}, \ldots, x_{r}\right) T^{r-k} .
$$

Now $\left|\alpha_{s}^{e_{u}}\right|=q_{v}^{e_{u} / r}$ for any $1 \leq s \leq r$ by Proposition 2.6. For any $0 \leq k \leq r$, we obtain

$$
\begin{aligned}
\left|S_{k}\left(\alpha_{1}^{e_{u}}, \ldots, \alpha_{r}^{e_{u}}\right)-S_{k}\left(\pi_{v}^{j_{1}}, \ldots, \pi_{v}^{j_{r}}\right)\right| & \leq \max _{1 \leq s_{1}<\cdots<s_{k} \leq r}\left\{q_{v}^{k \cdot e_{u} / r}, q_{v}^{j_{s_{1}}+\cdots+j_{s_{k}}}\right\} \\
& \leq q_{v}^{k \cdot e_{u}} \\
& \leq q_{v}^{r \cdot e_{u}}=q^{r \cdot e_{u} \cdot f_{v \mid \pi_{0}}}
\end{aligned}
$$

since $j_{s} \leq e_{u}$ for each $s$ by Proposition 3.4. Since $e_{u}$ divides $e_{u \mid \pi} \cdot C_{1}(q, r)$ and both $e_{u \mid \pi}$ and $f_{v \mid \pi_{0}}$ are less than or equal to $n_{K}=[K: F]$, we see that

$$
q^{r \cdot e_{u} \cdot f_{v \mid \pi_{0}}} \leq q^{C_{2}}<q^{\operatorname{deg}(\pi)}=|\pi|,
$$

which means that all absolute values of coefficients of $P_{v, e_{u}}(T)-\prod_{s=1}^{r}\left(T-\pi_{v}^{j_{s}}\right)$ are smaller than $|\pi|$. Therefore the congruence (3.3) implies $P_{v, e_{u}}(T)=\prod_{s=1}^{r}\left(T-\pi_{v}^{j_{s}}\right)$. Comparing the absolute values of the roots of $P_{v, e_{u}}(T)$ and $\prod_{s=1}^{r}\left(T-\pi_{v}^{j_{s}}\right)$, we see that $e_{u} / r=j_{s}$ for any $1 \leq s \leq r$, which implies the conclusion.

Set $e_{\phi}:=\operatorname{gcd}\left\{e_{u} ; u \mid \pi\right\}$ and $\mathbb{S}_{r}:=\left\{\mathbf{s}=\left(s_{1}, \ldots, s_{r}\right) \in \mathbb{Z}^{r} ; 1 \leq s_{k} \leq r\right\}$.

LeMma 3.6. Suppose that $\operatorname{deg}(\pi)>C_{2}$.

(1) $e_{\phi} \mid n_{K} \cdot C_{1}(q, r)$. If $\pi$ is unramified in $K_{\mathrm{s}}$, then $e_{\phi} \mid[K: F]_{\mathrm{i}} \cdot C_{1}(q, r)$.

(2) For any $\left(s_{1}, \ldots, s_{r}\right) \in \mathbb{S}_{r}$, the relation $e_{\phi} \cdot\left(i_{s_{1}}+\cdots+i_{s_{r}}-1\right) \equiv 0\left(\bmod q_{\pi}-1\right)$ holds.

Proof. Let $u$ be a finite place of $K$ above $\pi$ and $u_{0}$ the place of $K_{\mathrm{s}}$ below $u$. Then $e_{u \mid \pi}=e_{u_{0} \mid \pi}[K: F]_{\mathrm{i}}$ since $u_{0}$ is totally ramified in $K$ if $K \neq K_{\mathrm{s}}$. Hence (1) follows from the relation $e_{u} \mid e_{u \mid \pi} C_{1}(q, r)$. By Proposition 3.5, we see that $i_{s} \cdot e_{\phi} \equiv e_{\phi} / r\left(\bmod q_{\pi}-1\right)$. Adding these congruences for $s_{1}, \ldots, s_{r}$ gives

$$
e_{\phi} \cdot\left(i_{s_{1}}+\cdots+i_{s_{r}}\right) \equiv e_{\phi} \quad\left(\bmod q_{\pi}-1\right),
$$

which proves (2).

There exist only finitely many places of $F$ which are ramified in $K_{\mathrm{s}}$. Define $C_{3}=C_{3}\left(K_{\mathrm{s}}\right)$ to be the maximal degree of such places and set

$$
C_{4}=C_{4}\left(n_{K}, q, r, K_{\mathrm{S}}\right):=\max \left\{C_{2}\left(n_{K}, q, r\right), C_{3}\left(K_{\mathrm{S}}\right)\right\} .
$$

TheOREM 3.7. Suppose that $r=p^{v}>1$ and $r$ does not divide $[K: F]_{\mathrm{i}}$. If $\operatorname{deg}(\pi)>C_{4}$, then the set $\mathscr{D}(K, r, \pi)$ is empty. 
Proof. Assume that $[\phi] \in \mathscr{D}(K, r, \pi)$ and $\operatorname{deg}(\pi)>C_{4}$. Then $\pi$ is unramified in $K_{\mathrm{s}}$ by $\operatorname{deg}(\pi)>C_{3}$. Proposition 3.5 and Lemma 3.6 imply that $r$ divides $[K: F]_{\mathrm{i}} \cdot C_{1}(q, r)$. The integer $C_{1}(q, r)$ is prime to $p$ and so $r$ must divide $[K: F]_{\mathrm{i}}$, which contradicts the assumption on $r$.

Remark 3.8. In Section 5, we see that, if $r$ divides $[K: F]_{\mathrm{i}}$, then $\mathscr{D}(K, r, \pi)$ is not empty.

\section{Observations at places with small degree}

In this section, using Propositions 4.6 and 4.8, we prove Theorem 4.9 on the emptiness of $\mathscr{D}(K, r, \pi)$ when $r$ is not a $p$-power. In this case, we also prove its uniform version (Theorem 4.10).

\subsection{The effective Chebotarev density theorem}

Recall some basic facts on function field arithmetic. Let $L$ be an algebraic extension of $K$. The constant field $\mathbb{F}_{L}$ of $L$ is the algebraic closure of $\mathbb{F}_{q}$ in $L$. If $L=\mathbb{F}_{L} K$, then $L$ is called a constant field extension of $K$, which is unramified at any places [Ro, Proposition 8.5]. If $\mathbb{F}_{L}=\mathbb{F}_{K}$, then $L$ is called a geometric extension of $K$. In general, the field $\mathbb{F}_{L} K$ is the maximal constant extension of $K$ in $L$ and the extension $L / \mathbb{F}_{L} K$ is geometric. Set $[L: K]_{\mathrm{g}}:=\left[L: \mathbb{F}_{L} K\right]$ if $L / K$ is finite, which is called the geometric extension degree of $L / K$. For example, for any $a \in A$, the field $F(\mathcal{C}[a])$ arising from the Carlitz module is a geometric extension of $F$.

Denote by $\operatorname{Div}(K)$ the divisor group of $K$, that is, the free abelian group generated by all places of $K$. We write divisors additively, so that a typical divisor is of the form $D=\sum_{v} n_{v} v$. The notation $v \notin D$ means that $n_{v}=0$. The degree of a place $v$ of $K$ is defined by $\operatorname{deg}_{K} v:=$ $\left[\mathbb{F}_{v}: \mathbb{F}_{K}\right]$ and it is extended to any divisor $D=\sum_{v} n_{v} v$ by $\operatorname{deg}_{K} D=\sum_{v} n_{v} \operatorname{deg}_{K} v$. The degree $\operatorname{deg}_{F} \pi$ of a finite place $\pi$ of $F$ is exactly the degree $\operatorname{deg}(\pi)$ as a polynomial.

Suppose that $L$ is a finite separable extension of $K$. Then the conorm map $i_{L / K}$ : $\operatorname{Div}(K) \rightarrow \operatorname{Div}(L)$ is defined to be the linear extension of

$$
i_{L / K} v=\sum_{w \mid v} e_{w \mid v} w,
$$

where $v$ is a place of $K$. The following is known (cf. [Ro, Proposition 7.7]).

Lemma 4.1. Let $w$ be a place of $L$ above a place $v$ of $K$ and $D \in \operatorname{Div}(K)$. Then

$$
\operatorname{deg}_{L} i_{L / K} D=[L: K]_{\mathrm{g}} \operatorname{deg}_{K} D \quad \text { and } \quad \operatorname{deg}_{L} w=\frac{f_{w \mid v}}{\left[\mathbb{F}_{L}: \mathbb{F}_{K}\right]} \operatorname{deg}_{K} v .
$$

For any place $w$ of $L$ above a place $v$ of $K$, denote by $\mathfrak{p}_{w}$ the maximal ideal of $\mathcal{O}_{L_{w}}$ and let $\delta_{w}$ be the exact power of $\mathfrak{p}_{w}$ dividing the different of $\mathcal{O}_{L_{w}}$ over $\mathcal{O}_{K_{v}}$. Then it satisfies $\delta_{w} \geq e_{w \mid v}-1$ with equality holding if and only if $p$ does not divide $e_{w \mid v}$. Define the ramification divisor of $L / K$ by $\mathcal{D}_{L / K}=\sum_{w} \delta_{w} w$. For any intermediate field $K^{\prime}$ of $L / K$, we see that

$$
\mathcal{D}_{L / K}=\mathcal{D}_{L / K^{\prime}}+i_{L / K^{\prime}} \mathcal{D}_{K^{\prime} / K}
$$

(for example, see [Se2, Ch. III 4]). Hence $\mathcal{D}_{L / K^{\prime}} \leq \mathcal{D}_{L / K}$ holds. In addition, the following holds (cf. [CL, Lemma 2.6]). 
Lemma 4.2. Let $L / K$ and $L^{\prime} / K$ be finite separable extensions. Then

$$
\mathcal{D}_{L L^{\prime} / K} \leq i_{L L^{\prime} / L} \mathcal{D}_{L / K}+i_{L L^{\prime} / L^{\prime}} \mathcal{D}_{L^{\prime} / K}
$$

Now let $E$ be a finite Galois extension of $K$ and $v$ a place of $K$ unramified in $E$. For any place $w$ of $E$ above $v$, denote by $\operatorname{Fr}_{w \mid v}$ the Frobenius element in $\operatorname{Gal}(E / K)$. Then the conjugacy class

$$
\left[\frac{E / K}{v}\right]:=\left\{\operatorname{Fr}_{w \mid v} ; w \mid v\right\}
$$

in $\operatorname{Gal}(E / K)$ consists of these elements. Define $\Sigma_{E / K}$ to be the divisor of $K$ that is the sum of all ramified places of $K$ in $E$. As a consequence of the effective version of the Chebotarev density theorem [KS, Theorem 1], the following holds.

Proposition 4.3. (Chen and Lee [CL, Corollary 3.4]) Let $E / K$ be a finite Galois extension and $\Sigma$ a divisor of $K$ such that $\Sigma \geq \Sigma_{E / K}$. Set $d_{0}:=\left[\mathbb{F}_{K}: \mathbb{F}_{q}\right]$ and $d:=\left[\mathbb{F}_{E}: \mathbb{F}_{K}\right]$. Define the constant $B=B(E / K, \Sigma)$ by

$$
B=\max \left\{\operatorname{deg}_{K} \Sigma, \operatorname{deg}_{E} \mathcal{D}_{E / \mathbb{F}_{E} K}, 2\left[E: \mathbb{F}_{E} K\right]-2,1\right\} .
$$

Then for any non-empty conjugacy class $\mathscr{C}$ in $\mathrm{Gal}(E / K)$, there exists a place $v$ of $K$ with $v \notin \Sigma$ such that:

- $\mathscr{C}=\left[\frac{E / K}{v}\right]$

- $\operatorname{deg}_{K} v \leq \frac{4}{d_{0}} \log _{q} \frac{4}{3}\left(B+3 g_{K}+3\right)+d$,

where $g_{K}$ is the genus of $K$.

Let $\pi$ be a monic irreducible element of $A$ and $m \geq 1$ an integer which divides $\# \mathbb{F}_{\pi}^{\times}=$ $q_{\pi}-1$. A monic irreducible element $\pi_{0}$ distinct from $\pi$ is called an mth power residue modulo $\pi$ if $\left(\pi_{0} \bmod \pi\right) \in\left(\mathbb{F}_{\pi}^{\times}\right)^{m}$. As an application of Proposition 4.3, we show that one can find an $m$ th power residue modulo $\pi$ whose degree is smaller than $\operatorname{deg}(\pi)$ if $\operatorname{deg}(\pi)$ is sufficiently large. Denote by $F_{m}$ the unique subfield of $F(\mathcal{C}[\pi])$ with $\left[F_{m}: F\right]=m$ and consider the character $\chi(m): G_{F} \stackrel{\chi_{\pi}}{\longrightarrow} \mathbb{F}_{\pi}^{\times} \rightarrow \mathbb{F}_{\pi}^{\times} /\left(\mathbb{F}_{\pi}^{\times}\right)^{m}$.

LEMMA 4.4. The following are equivalent:

(1) $\pi_{0}$ is an mth power residue modulo $\pi$;

(2) $\chi(m)\left(\right.$ Frob $\left._{\pi_{0}}\right)=1$;

(3) Frob $\left._{\pi_{0}}\right|_{F_{m}}=$ id.

Proof. It is trivial when $m=1$. If not, then this lemma immediately follows from the facts that $\chi_{\pi}\left(\operatorname{Frob}_{\pi_{0}}\right) \equiv \pi_{0}(\bmod \pi)$ and $F_{m}$ is the fixed subfield of $F^{\text {sep }}$ by $\operatorname{Ker}(\chi(m))$.

Denote by $\tilde{K}$ the Galois closure of $K_{\mathrm{s}}$ over $F$. Set $E:=\tilde{K} F_{m}$, which is also a Galois extension of $F$. Consider the divisor $\Sigma:=\Sigma_{E / F}+\pi+\infty \in \operatorname{Div}(F)$. For the constant $B=$ $B(E / F, \Sigma)=\max \left\{\operatorname{deg}_{F} \Sigma, \operatorname{deg}_{E} \mathcal{D}_{E / \mathbb{F}_{E} F}, 2\left[E: \mathbb{F}_{E} F\right]-2,1\right\}$, we obtain the following estimate. 
Lemma 4.5. Let $n$ be a positive integer. Then there exists a constant $C_{5}=$ $C_{5}\left(K_{\mathrm{s}}, n_{K}, q, m, n\right)>0$ such that for any $\pi$ satisfying $\operatorname{deg}(\pi)>C_{5}$, the inequality

$$
4 \log _{q} \frac{4}{3}(B+3)+\left[\mathbb{F}_{\tilde{K}}: \mathbb{F}_{q}\right]<\frac{1}{n} \operatorname{deg}(\pi)
$$

holds.

Proof. We first compute an upper bound of $B$. We may assume that $\pi$ is unramified in $K_{\mathrm{S}}$. Since the degree $[\tilde{K}: F]$ is less than or equal to $n_{K}$ !, we see that $\left[\mathbb{F}_{\tilde{K}}: \mathbb{F}_{q}\right] \leq n_{K}$ ! and $\left[E: \mathbb{F}_{E} F\right] \leq m \cdot n_{K}$ !. By Example 2.7, the infinite place $\infty$ of $F$ is split into at most $m$ places in $F_{m}$ whose ramification indices divide $q-1$ and $\pi$ is totally ramified or unramified (if $m=1$ ) in $F_{m}$. Thus we see that

$$
\operatorname{deg}_{F} \Sigma \leq \operatorname{deg}_{F}\left(\Sigma_{F_{m} / F}+\Sigma_{\tilde{K} / F}+\pi+\infty\right) \leq 2 \operatorname{deg}(\pi)+2+\operatorname{deg}_{F} \Sigma_{\tilde{K} / F} .
$$

Now $\mathcal{D}_{E / \mathbb{F}_{E} F}=\mathcal{D}_{E / F}$ holds. Lemmas 4.1 and 4.2 imply that

$$
\begin{aligned}
\operatorname{deg}_{E} \mathcal{D}_{E / F} & \leq \operatorname{deg}_{E} i_{E / \tilde{K}} \mathcal{D}_{\tilde{K} / F}+\operatorname{deg}_{E} i_{E / F_{m}} \mathcal{D}_{F_{m} / F} \\
& \leq m \cdot \operatorname{deg}_{\tilde{K}} \mathcal{D}_{\tilde{K} / F}+\left[E: F_{m}\right]_{\mathrm{g}} \cdot \operatorname{deg}_{F_{m}}\left(\sum_{v \mid \infty}(q-2) v+m \pi\right) \\
& \leq m \cdot \operatorname{deg}_{\tilde{K}} \mathcal{D}_{\tilde{K} / F}+n_{K} ! \cdot m(q-2+\operatorname{deg}(\pi)) .
\end{aligned}
$$

Hence there exist positive constants $B_{1}$ and $B_{2}$ depending only on $K_{\mathrm{s}}, n_{K}, q$ and $m$ such that $B \leq B_{1} \cdot \operatorname{deg}(\pi)+B_{2}$ holds. Therefore if $\operatorname{deg}(\pi)$ is sufficiently large, then $4 \log _{q} \frac{4}{3}(B+3)$ $+\left[\mathbb{F}_{\tilde{K}}: \mathbb{F}_{q}\right]<(1 / n) \operatorname{deg}(\pi)$ holds.

Proposition 4.3 and Lemma 4.5 imply the following.

Proposition 4.6. Let $n$ be a positive integer. If $\operatorname{deg}(\pi)>C_{5}$, then there exist a monic irreducible element $\pi_{0} \in A$ and a place $v$ of $K$ above $\pi_{0}$ such that:

(1) $\pi_{0}$ is an mth power residue modulo $\pi$;

(2) $\operatorname{deg}\left(\pi_{0}\right)<(1 / n) \operatorname{deg}(\pi)$;

(3) $f_{v \mid \pi_{0}}=1$.

Proof. We may assume that $K=K_{\mathrm{S}}$ since the extension $K / K_{\mathrm{S}}$ is totally ramified at any place if $K \neq K_{\mathrm{s}}$. Let $\tilde{K}$ and $E=\tilde{K} F_{m}$ be as above and fix an element $\sigma \in \operatorname{Gal}(E / F)$ such that $\left.\sigma\right|_{K F_{m}}=$ id. For the conjugacy class $\mathscr{C}$ of $\sigma$ in $\operatorname{Gal}(E / F)$, by Proposition 4.3 and Lemma 4.5 , there exists a place $\pi_{0}$ of $F$ with $\pi_{0} \notin \Sigma$ (hence it is a finite place) such that $\left[(E / F) / \pi_{0}\right]=\mathscr{C}$ and $\operatorname{deg}\left(\pi_{0}\right)<(1 / n) \operatorname{deg}(\pi)$, so that $\sigma=\mathrm{Fr}_{w \mid \pi_{0}}$ for some place $w$ of $E$. Then the decomposition group $Z_{w}$ of $w$ over $\pi_{0}$ is generated by $\sigma$ and it is a subgroup of $\operatorname{Gal}\left(E / K F_{m}\right)$. Denote by $K^{\prime}$ the fixed subfield of $E$ by $Z_{w}$. Then the place $v^{\prime}$ of $K^{\prime}$ below $w$ satisfies $e_{v^{\prime} \mid \pi_{0}}=1$ and $f_{v^{\prime} \mid \pi_{0}}=1$. Hence $f_{v \mid \pi_{0}}=1$, where $v$ is the place of $K$ below $v^{\prime}$. By construction, we see that Frob $_{v} \mid F_{m}=$ id. Lemma 4.4 means that $\pi_{0}$ is an $m$ th power residue modulo $\pi$. 


\subsection{Non-p-power rank case}

Let $\phi$ be a rank- $r$ Drinfeld module over $K$ satisfying $[\phi] \in \mathscr{D}(K, r, \pi)$. In this subsection, we always assume that $r=r_{0} \cdot p^{v}$ for some $r_{0}>1$ which is prime to $p$. Now let $i_{1}, \ldots, i_{r}$ be the positive integers satisfying $\bar{\rho}_{\phi, \pi}^{\mathrm{ss}} \simeq \chi_{\pi}^{i_{1}} \oplus \cdots \oplus \chi_{\pi}^{i_{r}}$ by (D2). For any $\mathbf{s}=\left(s_{1}, \ldots, s_{r}\right) \in \mathbb{S}_{r}$, set $\varepsilon_{\mathbf{s}}:=\chi_{\pi}^{i_{s_{1}}+\cdots+i_{s_{r}}-1}$ and define

$$
\epsilon:=\left(\varepsilon_{\mathbf{S}}\right)_{\mathbf{s} \in \mathbb{S}_{r}}: G_{F} \rightarrow\left(\mathbb{F}_{\pi}^{\times}\right)^{\oplus r^{r}} .
$$

Set $m_{\phi}:=\# \epsilon\left(G_{F}\right)$, which is the least common multiple of the orders of $\varepsilon_{\mathbf{s}}$. Since $\epsilon$ factors through $\mathbb{F}_{\pi}^{\times}$, the image $\epsilon\left(G_{F}\right)$ is cyclic and $m_{\phi} \mid\left(q_{\pi}-1\right)$. Then we obtain the following commutative diagram.

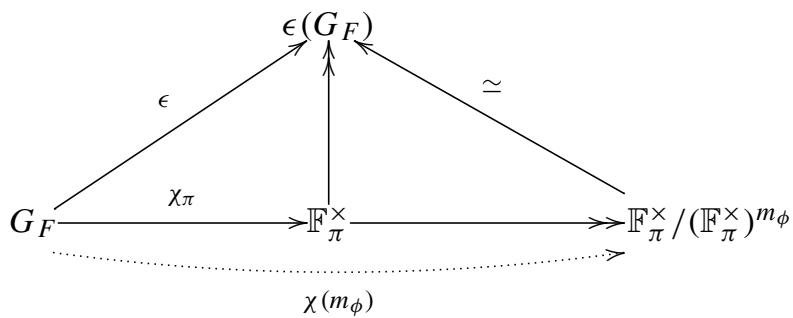

Hence a monic irreducible element $\pi_{0}$ is an $m_{\phi}$ th power residue modulo $\pi$ if and only if $\varepsilon_{\mathbf{s}}\left(\right.$ Frob $\left._{\pi_{0}}\right)=1$ for any $\mathbf{s} \in \mathbb{S}_{r}$.

LEMMA 4.7. If $\operatorname{deg}(\pi)>C_{2}\left(n_{K}, q, r\right)$, then $m_{\phi}$ divides the greatest common divisor $\left(e_{\phi}, q_{\pi}-1\right)$. In particular, it divides $n_{K} C_{1}(q, r)$.

Proof. It follows from Lemma 3.6(2).

PROPOSITION 4.8. If there exist a monic irreducible element $\pi_{0}$ and a finite place $v$ of $K$ above $\pi_{0}$ such that $\operatorname{deg}(\pi)>f_{v \mid \pi_{0}} \operatorname{deg}\left(\pi_{0}\right)$ and $r_{0}$ does not divide $f_{v \mid \pi_{0}}$, then $m_{\phi}>1$ and $\chi\left(m_{\phi}\right)\left(\operatorname{Frob}_{v}\right) \neq 1$.

Proof. Assume that either $m_{\phi}=1$ or $\chi\left(m_{\phi}\right)\left(\operatorname{Frob}_{v}\right)=1$ holds. Then $\varepsilon_{\mathbf{s}}\left(\operatorname{Frob}_{v}\right)=1$ for any $\mathbf{s} \in \mathbb{S}_{r}$. Denote by $a_{v, p^{v}} \in A$ the coefficient of $T^{r-p^{v}}$ in the characteristic polynomial $P_{v}(T)$ of Frob $v$ on $T_{\pi}(\phi)$. It is given by $a_{v, p^{v}}=(-1)^{p^{v}} S_{p^{v}}\left(\alpha_{1}, \ldots, \alpha_{r}\right)$, where $\alpha_{1}, \ldots, \alpha_{r}$ are the roots of $P_{v}(T)$ and $S_{p^{v}}\left(x_{1}, \ldots, x_{r}\right)$ is the fundamental symmetric polynomial of degree $p^{v}$ with $r$ variables. We regard the product $\mathbb{S}_{p^{v}}^{r_{0}}$ of $\mathbb{S}_{p^{v}}$ as a subset of $\mathbb{S}_{r}$. Consider the subset $\mathbb{S}_{r, p^{v}}:=\left\{\left(s_{1}, \ldots, s_{p^{v}}\right) ; 1 \leq s_{1}<\cdots<s_{p^{v}} \leq r\right\}$ of $\mathbb{Z}^{p^{v}}$. Since $S_{p^{v}}\left(x_{1}, \ldots, x_{r}\right)$ is the sum of $\left(\begin{array}{c}r \\ p^{v}\end{array}\right)$ monomials of degree $p^{v}$, we obtain that

$$
\begin{aligned}
\left(a_{v, p^{v}}\right)^{r_{0}} & =(-1)^{r} S_{p^{v}}\left(\alpha_{1}, \ldots, \alpha_{r}\right)^{r_{0}} \\
& \equiv(-1)^{r}\left(\sum_{\left(s_{1}, \ldots, s_{p^{v}}\right) \in \mathbb{S}_{r, p^{v}}} \chi_{\pi}^{i_{s_{1}}+\cdots+i_{s} p^{v}}\left(\operatorname{Frob}_{v}\right)\right)^{r_{0}} \\
& \equiv(-1)^{r} \sum_{\mathbf{s} \in \mathbb{S}_{p^{\nu}}^{r_{0}}} \varepsilon_{\mathbf{s}}\left(\operatorname{Frob}_{v}\right) \chi_{\pi}\left(\operatorname{Frob}_{v}\right)
\end{aligned}
$$




$$
\begin{aligned}
& \equiv(-1)^{r} \sum_{\mathbf{s} \in \mathbb{S}_{p^{v}}^{r_{0}}} \chi_{\pi}\left(\text { Frob }_{v}\right) \\
& \equiv(-1)^{r}\left(\begin{array}{c}
r \\
p^{v}
\end{array}\right)^{r_{0}} \pi_{0}^{f_{v \mid \pi_{0}}} \quad(\bmod \pi) .
\end{aligned}
$$

Since $\left(\begin{array}{c}r \\ p^{v}\end{array}\right)$ is not divisible by $p$, we see that $(-1)^{r}\left(\begin{array}{c}r \\ p^{v}\end{array}\right)^{r_{0}} \pi_{0}^{f_{v \mid \pi_{0}}} \not \equiv 0(\bmod \pi)$. Here

$$
\left|\left(a_{v, p^{v}}\right)^{r_{0}}\right| \leq q_{v}=q^{f_{v \mid \pi_{0}} \operatorname{deg}\left(\pi_{0}\right)}<|\pi| \quad \text { and } \quad\left|(-1)^{r}\left(\begin{array}{c}
r \\
p^{v}
\end{array}\right)^{r_{0}} \pi_{0}^{f_{v \mid \pi_{0}}}\right|=\left|\pi_{0}^{f_{v \mid \pi_{0}}}\right|=q_{v}<|\pi| .
$$

Hence the above congruence implies that $\left(a_{v, p^{v}}\right)^{r_{0}}=(-1)^{r}\left(\begin{array}{c}r \\ p^{v}\end{array}\right)^{r_{0}} \pi_{0}^{f_{v \mid \pi_{0}}}$. Comparing the $\pi_{0^{-}}$ adic valuations of both sides, we obtain $r_{0} \mid f_{v \mid \pi_{0}}$, which is a contradiction.

Set

$$
\begin{aligned}
& C_{6}=C_{6}\left(K_{\mathrm{s}}, n_{K}, q, r\right):=\max \left\{C_{5}\left(K_{\mathrm{s}}, n_{K}, q, m, 1\right) ; m \mid n_{K} C_{1}(q, r)\right\} \\
& C_{7}=C_{7}\left(K_{\mathrm{s}}, n_{K}, q, r\right):=\max \left\{C_{2}\left(n_{K}, q, r\right), C_{6}\left(K_{\mathrm{s}}, n_{K}, q, r\right)\right\} .
\end{aligned}
$$

Then we have the following theorem.

THEOREM 4.9. Suppose that $r=r_{0} p^{v}$ as above and $\operatorname{deg}(\pi)>C_{7}$. Then the set $\mathscr{D}(K, r, \pi)$ is empty.

Proof. Assume that $\mathscr{D}(K, r, \pi)$ is not empty and $[\phi] \in \mathscr{D}(K, r, \pi)$. By Proposition 4.6, there exist a monic irreducible element $\pi_{0}$ and a place $v$ of $K$ above $\pi_{0}$ such that $f_{v \mid \pi_{0}}=1$, $\operatorname{deg}\left(\pi_{0}\right)<\operatorname{deg}(\pi)$ and $\chi\left(m_{\phi}\right)\left(\right.$ Frob $\left._{\pi_{0}}\right)=1$. However, since $\pi_{0}$ and $v$ satisfy the assumptions of Proposition 4.8, we see that $\chi\left(m_{\phi}\right)\left(\right.$ Frob $\left._{v}\right)=\chi\left(m_{\phi}\right)\left(\right.$ Frob $\left._{\pi_{0}}\right) \neq 1$.

By the same argument, we can also prove a uniform version. For a fixed finite separable extension $K_{0}$ of $F$ with degree $n_{0}:=\left[K_{0}: F\right]$ and a positive integer $n$, set

$$
C_{8}=C_{8}\left(K_{0}, q, r, n\right):=\max \left\{C_{2}\left(n n_{0}, q, r\right), \max \left\{C_{5}\left(K_{0}, n_{0}, q, m, n\right) ; m \mid n_{0} C_{1}(q, r)\right\}\right\} .
$$

THEOREM 4.10. Let $r=r_{0} p^{\nu}, K_{0}$, and $n$ be as above. Suppose that $r_{0}$ does not divide $n$. If $\operatorname{deg}(\pi)>C_{8}$, then for any finite extension $K$ of $K_{0}$ satisfying $\left[K: K_{0}\right]=n$, the set $\mathscr{D}(K, r, \pi)$ is empty, namely the union

$$
\bigcup_{\left[K: K_{0}\right]=n} \mathscr{D}(K, r, \pi)
$$

is empty.

Proof. Let $K$ be a finite extension of $K_{0}$ with $\left[K: K_{0}\right]=n$ and assume that $[\phi] \epsilon$ $\mathscr{D}(K, r, \pi)$. Applying Proposition 4.6 to $K_{0}$, we can find a monic irreducible element $\pi_{0}$ and a finite place $v_{0}$ of $K_{0}$ above $\pi_{0}$ such that $f_{v_{0} \mid \pi_{0}}=1, n \operatorname{deg}\left(\pi_{0}\right)<\operatorname{deg}(\pi)$ and $\chi\left(m_{\phi}\right)\left(\right.$ Frob $\left._{\pi_{0}}\right)=1$. Now we can take a place $v$ of $K$ above $v_{0}$ such that $f_{v \mid v_{0}}\left(=f_{v \mid \pi_{0}}\right)$ is not divisible by $r_{0}$. Indeed, if not, then $r_{0}$ must divide $n=\sum_{v \mid v_{0}} e_{v \mid v_{0}} f_{v \mid v_{0}}$. Since $f_{v \mid \pi_{0}} \operatorname{deg}\left(\pi_{0}\right)<$ $n \operatorname{deg}\left(\pi_{0}\right)<\operatorname{deg}(\pi)$ by Proposition 4.8, we see that $\chi\left(m_{\phi}\right)\left(\operatorname{Frob}_{v}\right)=\chi\left(m_{\phi}\right)\left(\operatorname{Frob}_{\pi_{0}}\right)^{f_{v \mid \pi_{0}}}$ $\neq 1$. This is a contradiction. 


\section{Comparison with number field case}

In this final section, we compare the Rasmussen-Tamagawa conjecture and its Drinfeld module analogue. After studying the similarity between them, we construct an example of a Drinfeld module satisfying Rasmussen-Tamagawa type conditions for any $\pi$ and prove Theorem 5.6. We also prove the infiniteness of $\mathscr{D}(K, r, \pi)$ for $r \geq 2$ and $\pi=t$ in Proposition 5.15

\subsection{Defining conditions of $\mathscr{D}(K, r, \pi)$}

In the number field case, $\mathscr{A}(k, g, \ell)$ is defined by the equivalent conditions (RT-1), (RT-2), and (RT-3) in Section 1. The equivalence of them follows from the criterion of Néron-OggShafarevich and the next group theoretic lemma.

LEMma 5.1. (Rasmussen and Tamagawa [RT2, Lemma 3.4]) Let $\mathbb{F}$ be a finite field of characteristic $\ell$. Suppose $G$ is a profinite group, $N \subset G$ is a pro- $\ell$ open normal subgroup, and $C=G / N$ is a finite cyclic subgroup with $\# C \mid \# \mathbb{F}^{\times}$. Let $V$ be an $\mathbb{F}$-vector space of dimension $r$ on which $G$ acts continuously. Fix a group homomorphism $\chi_{0}: G \rightarrow \mathbb{F}^{\times}$with $\operatorname{Ker}\left(\chi_{0}\right)=N$.

Then there exists a filtration

$$
0=V_{0} \subset V_{1} \subset \cdots \subset V_{r}=V
$$

such that each $V_{s}$ is $G$-stable and $\operatorname{dim}_{\mathbb{F}} V_{s}=s$ for any $0 \leq s \leq r$. Moreover, for each $1 \leq$ $s \leq r$, the $G$-action on each quotient $V_{s} / V_{s-1}$ is given by $\chi_{0}^{i_{s}}$ for some integer $i_{s}$ satisfying $0 \leq i_{s}<\# C$.

Remark 5.2. In [RT2], this lemma is proved when $\mathbb{F}=\mathbb{F}_{\ell}$. The general case can be proved in the same way.

As an analogue, we give two conditions which are equivalent to (D1)+(D2). Let $\phi$ be a rank- $r$ Drinfeld module over $K$ and let $\pi \in A$ be a monic irreducible element. Consider the field $K\left(\phi\left[\pi^{\infty}\right]\right):=K\left(\bigcup_{n \geq 1} \phi\left[\pi^{n}\right]\right)$ generated by all $\pi$-power torsion points of $\phi$. Then it coincides with the fixed subfield of $K^{\text {sep }}$ by the kernel of $\rho_{\phi, \pi}: G_{K} \rightarrow \operatorname{GL}_{r}\left(A_{\pi}\right)$. Recall that the mod $\pi$ Carlitz character $\chi_{\pi}: G_{K} \rightarrow \operatorname{GL}_{\mathbb{F}_{\pi}}(\mathcal{C}[\pi]) \simeq \mathbb{F}_{\pi}^{\times}$is an analogue of the mod $\ell$ cyclotomic character. For the field $L:=K(\phi[\pi]) \cap K(\mathcal{C}[\pi])$, we can prove the next proposition in the same way as the abelian variety case.

PROPOSITION 5.3. The following conditions are equivalent.

(DR-1) $K\left(\phi\left[\pi^{\infty}\right]\right) / L$ is a pro- $p$ extension which is unramified at any finite place of $L$ not lying above $\pi$.

(DR-2) $\phi$ has good reduction at any finite place of $K$ not lying above $\pi$ and $K(\phi[\pi]) / L$ is a p-extension.

(DR-3) $\phi$ satisfies (D1) and (D2).

Remark 5.4. Unlike the abelian variety case, the field $K(\phi[\pi])$ may not contain $K(\mathcal{C}[\pi])$. For example, for $x \in \mathbb{F}_{q}^{\times} \backslash\{1\}$, consider the rank-one Drinfeld module $\phi$ over $F$ determined by $\phi_{t}=t+x \tau$ and suppose $q \neq 2$. Then the fields $F(\phi[t])$ and $F(\mathcal{C}[t])$ are generated by the roots of $t+x T^{q-1}$ and $t+T^{q-1}$, respectively. By Kummer theory, we see that $F(\phi[t]) \neq F(\mathcal{C}[t])$, so that $F(\phi[t]) \not \supset F(\mathcal{C}[t])$. 
Proof of Proposition 5.3. Since the kernel of $\mathrm{GL}_{r}\left(A_{\pi}\right) \rightarrow \mathrm{GL}_{r}\left(\mathbb{F}_{\pi}\right)$ is a pro- $p$ group, the extension $K\left(\phi\left[\pi^{\infty}\right]\right) / K(\phi[\pi])$ is always pro- $p$. The extension $K(\mathcal{C}[\pi]) / K$ is unramified at any finite place of $K$ not lying above $\pi$ (Example 2.7). Hence the conditions (DR-1) and (DR-2) are equivalent by Proposition 2.5. Suppose that (DR-2) holds. Since we have $[K(\phi[\pi]): L]=[M: K(\mathcal{C}[\pi])]$ for the composite field $M:=K(\phi[\pi]) K(\mathcal{C}[\pi])$, $M / K(\mathcal{C}[\pi])$ is also a $p$-extension. We regard $\chi_{\pi}$ as a character of $\operatorname{Gal}(M / K)$. Then the condition (DR-3) follows from Lemma 5.1 for $G=\operatorname{Gal}(M / K), \chi_{0}=\chi_{\pi}: G \rightarrow \mathbb{F}_{\pi}^{\times}, N=$ $\operatorname{Ker}\left(\chi_{0}\right)=\operatorname{Gal}(M / K(\mathcal{C}[\pi]))$, and $V=\phi[\pi]$. Conversely, if (DR-3) holds, then the image of $\operatorname{Gal}(K(\phi[\pi]) / L)$ by $\bar{\rho}_{\phi, \pi}$ is contained in

$$
\left\{\left(\begin{array}{cccc}
1 & * & \cdots & * \\
& 1 & \ddots & \vdots \\
& & \ddots & * \\
& & & 1
\end{array}\right) \in \mathrm{GL}_{r}\left(\mathbb{F}_{\pi}\right)\right\},
$$

which is a Sylow $p$-subgroup of $\mathrm{GL}_{r}\left(\mathbb{F}_{\pi}\right)$. Since $\bar{\rho}_{\phi, \pi}: \operatorname{Gal}(K(\phi[\pi]) / K) \rightarrow \mathrm{GL}_{r}\left(\mathbb{F}_{\pi}\right)$ is injective, we see that $K(\phi[\pi]) / L$ is a $p$-extension.

Remark 5.5. The original conjecture of Rasmussen and Tamagawa is formulated for abelian varieties of arbitrary dimension, and so we would like to formulate its function field analogue for some higher-dimensional objects (recall that Drinfeld modules are analogues of elliptic curves). In [An], Anderson introduced objects called $t$-motives as analogues of abelian varieties of higher dimensions, which are also generalizations of Drinfeld modules. In fact the category of Drinfeld modules is anti-equivalent to that of $t$-motives of dimension one. It is known that $t$-motives have the notions of good reduction and Galois representations attached to their $\pi$-torsion points (see, for example, [Ga1]), so that we can consider the conditions (D1) and (D2) for $t$-motives. Moreover, Proposition 5.3 is also generalized to $t$-motives since the Galois criterion of good reduction for $t$-motives holds. Therefore the set $\mathscr{M}(K, d, r, \pi)$ of isomorphism classes of $d$-dimensional $t$-motives over $K$ of rank $r$ satisfying the RasmussenTamagawa type conditions can be defined and the following question makes sense: Is the set $\mathscr{M}(K, d, r, \pi)$ empty for any $\pi$ with sufficiently large degree?

\subsection{Non-emptiness of $\mathscr{D}(K, r, \pi)$}

In this subsection, by constructing a concrete example, we prove the following theorem.

THEOREM 5.6. If $r$ divides $[K: F]_{\mathrm{i}}$, then the set $\mathscr{D}(K, r, \pi)$ is never empty for any $\pi$.

If $r=1$, then Theorem 5.6 is trivial since the Carlitz module $\mathcal{C}$ satisfies both (D1) and (D2). Assume that $r \geq 2$ and $[K: F]_{\mathrm{i}}$ is divisible by $r$. Then $r$ is a $p$-power and the $r$-power map $A \rightarrow A ; a \mapsto a^{r}$ is an injective ring homomorphism.

For any $a=\sum x_{n} t^{n} \in A$ with $x_{n} \in \mathbb{F}_{q}$, set $\hat{a}:=\sum x_{n}^{1 / r} t^{n}$. Then we see that $a \mapsto \hat{a}$ is a ring automorphism of $A$ and the map $A \rightarrow A ; a \mapsto \hat{a}^{r}$ is an injective $\mathbb{F}_{q}$-algebra homomorphism.

Lemma 5.7. Set $[K: F]_{\mathrm{i}}=p^{v}$. Then $K_{\mathrm{S}}=K^{p^{v}}$. 
Proof. Since $K$ is a purely inseparable extension of $K_{\mathrm{S}}$ of degree $p^{v}$, the field $K^{p^{v}}$ is contained in $K_{\mathrm{s}}$. Consider the sequence of fields $K \supset K^{p} \supset \cdots \supset K^{p^{v}}$. Proposition 7.4 of [Ro] implies that each extension $K^{p^{n}} / K^{p^{n+1}}$ is of degree $p$. Hence $\left[K: K^{p^{v}}\right]=p^{v}=[K$ : $K_{\mathrm{s}}$ ], which means that $K_{\mathrm{s}}=K^{p^{v}}$.

Since $r$ divides $[K: F]_{\mathrm{i}}$, Lemma 5.7 implies that $K$ contains the field $F^{1 / r}$. In particular the $r$ th root $t^{1 / r}$ of $t$ is contained in $K$. Then we have a new injective $A$-field structure $\iota: A \rightarrow K$ defined by $\iota(t)=t^{1 / r}$. Define the rank-one Drinfeld module

$$
\mathcal{C}^{\prime}: A \rightarrow K\{\tau\}
$$

over the $A$-field $(K, \iota)$ by $\mathcal{C}_{t}^{\prime}=t^{1 / r}+\tau$.

Set ${ }^{(r)} \mu:=\sum c_{i}^{r} \tau^{i}$ for any $\mu=\sum c_{i} \tau^{i} \in K\{\tau\}$. Then $K\{\tau\} \rightarrow K\{\tau\} ; \mu \mapsto^{(r)} \mu$ is a ring endomorphism. We can relate $\mathcal{C}^{\prime}$ with the Carlitz module $\mathcal{C}$ as follows.

LEMMA 5.8 .

(1) For any $a \in A,{ }^{(r)} \mathcal{C}_{\hat{a}}^{\prime}=\mathcal{C}_{a}$.

(2) For any element $\lambda \in \mathcal{C}^{\prime}[\hat{a}]$, there exists a unique $\delta \in \mathcal{C}[a]$ such that $\lambda=\delta^{1 / r}$.

Proof. Clearly ${ }^{(r)} \mathcal{C}_{\hat{x}}^{\prime}=x=\mathcal{C}_{x}$ for any $x \in \mathbb{F}_{q}$ and ${ }^{(r)} \mathcal{C}_{\hat{t}}^{\prime}={ }^{(r)} \mathcal{C}_{t}^{\prime}=\mathcal{C}_{t}$. Hence for any $a=$ $\sum x_{n} t^{n} \in A$,

$$
{ }^{(r)} \mathcal{C}_{\hat{a}}^{\prime}={ }^{(r)}\left(\sum x_{n}^{1 / r}\left(\mathcal{C}_{t}^{\prime}\right)^{n}\right)=\sum x_{n}\left(\mathcal{C}_{t}\right)^{n}=\mathcal{C}_{a} .
$$

For any $\lambda \in \mathcal{C}^{\prime}[\hat{a}]$, we see that

$$
0=\left(\mathcal{C}_{\hat{a}}^{\prime}(\lambda)\right)^{r}={ }^{(r)} \mathcal{C}_{\hat{a}}^{\prime}\left(\lambda^{r}\right)=\mathcal{C}_{a}\left(\lambda^{r}\right) .
$$

Hence $\lambda^{r} \in \mathcal{C}[a]$ and we have the injective homomorphism $\mathcal{C}^{\prime}[\hat{a}] \rightarrow \mathcal{C}[a] ; \lambda \mapsto \lambda^{r}$ of finite groups. Since $\# \mathcal{C}^{\prime}[\hat{a}]$ is equal to $\# \mathcal{C}[a]$ by $\operatorname{deg}(\hat{a})=\operatorname{deg}(a)$, it is a bijection.

Define $\Phi_{a}:=\mathcal{C}_{\hat{a}^{r}}^{\prime}=\left(\mathcal{C}_{\hat{a}}^{\prime}\right)^{r} \in K\{\tau\}$ for any $a \in A$. Then by construction it gives an $\mathbb{F}_{q^{-}}$ algebra homomorphism

$$
\Phi: A \rightarrow K\{\tau\},
$$

which is determined by $\Phi_{t}=\left(t^{1 / r}+\tau\right)^{r}$. Since $\iota\left(\hat{a}^{r}\right)=a$ holds, $\Phi$ is a rank-r Drinfeld module over $K$. Moreover it has good reduction at every finite place $v$ of $K$ since $v\left(t^{1 / r}\right) \geq 0$.

By the following proposition, we see that $[\Phi] \in \mathscr{D}(K, r, \pi)$, which implies Theorem 5.6.

PROPOSITION 5.9. Let $i$ be the positive integer satisfying $i r \equiv 1\left(\bmod q_{\pi}-1\right)$ and $i<$ $q_{\pi}-1$. Then the mod $\pi$ representation attached to $\Phi$ is of the form

$$
\bar{\rho}_{\Phi, \pi} \simeq\left(\begin{array}{cccc}
\chi_{\pi}^{i} & * & \cdots & * \\
& \chi_{\pi}^{i} & \ddots & \vdots \\
& & \ddots & * \\
& & & \chi_{\pi}^{i}
\end{array}\right) .
$$

Proof. It suffices to prove that $\bar{\rho}_{\Phi, \pi}^{\mathrm{ss}}=\left(\chi_{\pi}^{i}\right)^{\oplus r}$. For each $1 \leq s \leq r$, set

$$
V_{s}:=\left\{\lambda \in{ }_{\Phi} K^{\mathrm{sep}} ; \mathcal{C}_{\hat{\pi}^{s}}^{\prime}(\lambda)=0\right\} .
$$


For any $a \in A$ and $\lambda \in V_{s}$, we see that $\Phi_{a}(\lambda) \in V_{s}$ since $\mathcal{C}_{\hat{\pi}^{s}}^{\prime}\left(\Phi_{a}(\lambda)\right)=\mathcal{C}_{\hat{\pi}^{s}}^{\prime}\left(\mathcal{C}_{\hat{a}^{r}}^{\prime}(\lambda)\right)=$ $\mathcal{C}_{\hat{a}^{r}}^{\prime}\left(\mathcal{C}_{\hat{\pi}^{s}}^{\prime}(\lambda)\right)=0$. Hence $V_{s}$ is an $A$-submodule of ${ }_{\Phi} K^{\text {sep }}$ with the natural $G_{K}$-action. Moreover $\Phi_{\pi}(\lambda)=0$ for any $\lambda \in V_{s}$, so that $V_{s}$ is an $\mathbb{F}_{\pi}(=A / \pi A)$-vector space. Here $\Phi[\pi]=V_{r}$ by the definition of $\Phi$. Then we obtain the filtration

$$
0=V_{0} \subset V_{1} \subset V_{2} \subset \cdots \subset V_{r}=\Phi[\pi]
$$

of $G_{K}$-stable $\mathbb{F}_{\pi}$-subspaces of $\Phi[\pi]$. The map $V_{s} \rightarrow V_{1} ; \lambda \mapsto \mathcal{C}_{\hat{\pi}^{s-1}}^{\prime}(\lambda)$ induces a $G_{K^{-}}$ equivariant isomorphism $V_{s} / V_{s-1} \cong V_{1}$. Since $V_{1}=\mathcal{C}^{\prime}[\hat{\pi}]$ (as a set) and $\operatorname{deg}(\pi)=\operatorname{deg}(\hat{\pi})$, we have $\# V_{1}=q_{\hat{\pi}}=q_{\pi}\left(=\# \mathbb{F}_{\pi}\right)$. Hence $\operatorname{dim}_{\mathbb{F}_{\pi}} V_{1}=1$ and the semisimplification of $\Phi[\pi]$ (as an $\mathbb{F}_{\pi}\left[G_{K}\right]$-module) is $\Phi[\pi]^{\mathrm{ss}}=\bigoplus_{s=1}^{r} V_{s} / V_{s-1} \cong V_{1}^{\oplus r}$. For any $\sigma \in G_{K}$ and $\lambda \in V_{1}$, we prove $\sigma(\lambda)=\chi_{\pi}(\sigma)^{i} \cdot \lambda$ as follows. Take an element $a_{\sigma} \in A$ satisfying $a_{\sigma} \equiv \chi_{\pi}(\sigma)$ $(\bmod \pi)$. By Lemma 5.8(2), $\lambda=\delta^{1 / r}$ for some $\delta \in \mathcal{C}[\pi]$. Then

$$
\sigma(\lambda)^{r}=\sigma(\delta)=\chi_{\pi}(\sigma) \cdot \delta=\mathcal{C}_{a_{\sigma}}(\delta) .
$$

The $\mathbb{F}_{\pi}$-vector space structure of $V_{1}$ is determined by $\Phi$ and so $\chi_{\pi}(\sigma)^{i} \cdot \lambda=\Phi_{a_{\sigma}^{i}}(\lambda)=$ $\mathcal{C}_{\hat{a}_{\sigma}^{i r}}^{\prime}(\lambda)$. Since $i r \equiv 1\left(\bmod q_{\hat{\pi}}-1\right)$ holds, we have $\hat{a}_{\sigma}^{i r} \equiv \hat{a}_{\sigma}(\bmod \hat{\pi})$. This implies that $\mathcal{C}_{\hat{a}_{\sigma}^{i r}}^{\prime}(\lambda)=\mathcal{C}_{\hat{a}_{\sigma}}^{\prime}(\lambda)$. By Lemma $5.8(1)$, we obtain

$$
\left(\chi_{\pi}(\sigma)^{i} \cdot \lambda\right)^{r}=\left(\mathcal{C}_{\hat{a}_{\sigma}}^{\prime}(\lambda)\right)^{r}={ }^{(r)} \mathcal{C}_{\hat{a}_{\sigma}}^{\prime}\left(\lambda^{r}\right)=\mathcal{C}_{a_{\sigma}}(\delta)=\sigma(\lambda)^{r} .
$$

Since the $r$-power map is injective, we have $\sigma(\lambda)=\chi_{\pi}(\sigma)^{i} \cdot \lambda$. Hence the $G_{K}$-action on $V_{1}$ is given by $\chi_{\pi}^{i}$.

Remark 5.10. Let $u$ be a finite place of $K$ above $\pi$. Now $r$ divides $e_{u \mid \pi}$ by assumption. Set $e=e_{u \mid \pi} / r$. Since $i r \equiv 1\left(\bmod q_{\pi}-1\right)$, we see that

$$
\left.\chi_{\pi}^{i}\right|_{I_{K_{u}}}=\left(\omega_{1, K_{u}}\right)^{i \cdot e_{u \mid \pi}}=\left(\omega_{1, K_{u}}\right)^{i \cdot r \cdot e}=\left(\omega_{1, K_{u}}\right)^{e} .
$$

Hence the set of tame inertia weights of $\left.\bar{\rho}_{\Phi, \pi}\right|_{I_{K_{u}}}$ is $\mathrm{TI}_{K_{u}}\left(\bar{\rho}_{\Phi, \pi}\right)=\{e\}$.

\subsection{Infiniteness of $\mathscr{D}(K, r, t)$}

Finally, for $\pi=t$, we construct an infinite subset of $\mathscr{D}(K, r, t)$. In the number field case, the set $\mathscr{A}(k, g, \ell)$ is always finite because of the Shafarevich conjecture proved by Faltings [Fa], which states that there exist only finitely many isomorphism classes of abelian varieties over fixed $k$ with fixed dimension $g$ which have good reduction outside a fixed finite set of finite places of $k$. However, the Drinfeld module analogue of it does not hold.

Example 5.11. For any $a \in A$, consider the rank-two Drinfeld module $\phi^{(a)}: A \rightarrow F\{\tau\}$ given by $\phi_{t}^{(a)}=t+a \tau+\tau^{2}$. It is easily seen that $\phi^{(a)}$ has good reduction at any finite place of $F$. If $\phi^{(a)}$ is isomorphic to $\phi^{\left(a^{\prime}\right)}$ for some $a^{\prime} \in A$ over $F$, then there exists an element $c \in F$ such that $c \phi_{t}^{\left(a^{\prime}\right)}=\phi_{t}^{(a)} c$. Hence

$$
\phi_{t}^{\left(a^{\prime}\right)}=t+a^{\prime} \tau+\tau^{2}=t+c^{q-1} a \tau+c^{q^{2}-1} \tau^{2} .
$$

This means that $c \in \mathbb{F}_{q}^{\times}$and hence $a^{\prime}=c^{q-1} a=a$. Therefore the set of isomorphism classes $\left\{\left[\phi^{(a)}\right] ; a \in A\right\}$ is infinite. 
Let $W$ be a $G_{K}$-stable one-dimensional $\mathbb{F}_{q}$-vector space contained in $K^{\text {sep }}$ and write $\kappa_{W}: G_{K} \rightarrow \mathbb{F}_{q}^{\times}$for the character attached to $W$. Set $P_{W}(T):=\prod_{\lambda \in W}(T-\lambda)$, which is an $\mathbb{F}_{q}$-linear polynomial of the form

$$
P_{W}(T)=T^{q}+c_{W} T, \quad c_{W}:=\left(\prod_{\lambda \in W \backslash\{0\}}-\lambda\right) \in K^{\times}
$$

by [Go, Corollary 1.2.2]. For any $c \in K^{\times}$, denote by $\bar{c} \in K^{\times} /\left(K^{\times}\right)^{q-1}$ the class of $c$ and by $\kappa_{(c)}: G_{K} \rightarrow \mathbb{F}_{q}^{\times}$the character corresponding to $\bar{c}$ by the map $K^{\times} /\left(K^{\times}\right)^{q-1} \stackrel{\sim}{\rightarrow}$ $\operatorname{Hom}\left(G_{K}, \mathbb{F}_{q}^{\times}\right)$of Kummer theory.

LEMMA 5.12. For the above element $c_{W} \in K^{\times}$, the character $\kappa_{\left(-c_{W}\right)}$ coincides with $\kappa_{W}$.

Proof. Since $\lambda^{q-1}=-c_{W}$ for any $\lambda \in W \backslash\{0\}$, the character $\kappa_{\left(-c_{W}\right)}$ is given by $\kappa_{\left(-c_{W}\right)}(\sigma)=$ $\sigma(\lambda) / \lambda=\kappa_{W}(\sigma)$ for any $\sigma \in G_{K}$.

Identify $\mathbb{F}_{t}=A / t A=\mathbb{F}_{q}$. Then $\mathcal{C}[t]$ is a one-dimensional $\mathbb{F}_{q}$-subspace of $K^{\text {sep }}$ and $P_{\mathcal{C}[t]}(T)=T^{q}+t T$ by the definition of $\mathcal{C}$. By Lemma 5.12, we see that $\chi_{t}=\kappa_{(-t)}$. Note that $\chi_{t}^{i}=\kappa_{\left((-t)^{i}\right)}$ for any integer $i$.

Take $r$ elements $c_{1}, \ldots, c_{r} \in K^{\times}$. For any $1 \leq s \leq r$, define $f_{s}(\tau):=\left(\tau+c_{s}\right)(\tau+$ $\left.c_{s-1}\right) \cdots\left(\tau+c_{1}\right) \in K\{\tau\}$. Set $W_{s}:=\operatorname{Ker}\left(f_{s}: K^{\text {sep }} \rightarrow K^{\text {sep }}\right)$, which is a $G_{K}$-stable $s$-dimensional $\mathbb{F}_{q}$-subspace of $K^{\text {sep }}$. Thus we obtain the filtration

$$
0=W_{0} \subset W_{1} \subset \cdots \subset W_{r}
$$

of $\mathbb{F}_{q}\left[G_{K}\right]$-modules.

Lemma 5.13. The $\mathbb{F}_{q}$-linear representation $\bar{\rho}: G_{K} \rightarrow \mathrm{GL}_{\mathbb{F}_{q}}\left(W_{r}\right) \simeq \mathrm{GL}_{r}\left(\mathbb{F}_{q}\right)$ is of the form

$$
\bar{\rho} \simeq\left(\begin{array}{cccc}
\kappa_{\left(-c_{1}\right)} & * & \cdots & * \\
& \kappa_{\left(-c_{2}\right)} & \ddots & \vdots \\
& & \ddots & * \\
& & & \kappa_{\left(-c_{r}\right)}
\end{array}\right)
$$

Proof. For any $1 \leq s \leq r$, the quotient $W_{s} / W_{s-1}$ is isomorphic to $\operatorname{Ker}\left(\tau+c_{s}: K^{\text {sep }} \rightarrow K^{\text {sep }}\right)$ as an $\mathbb{F}_{q}\left[G_{K}\right]$-module. Hence each $W_{s} / W_{s-1}$ is embedded into $K^{\text {sep }}$. By Lemma 5.12 , the action of $G_{K}$ on $W_{s} / W_{s-1}$ is given by $\kappa_{\left(-c_{s}\right)}$.

Fix $r$ integers $i_{1}, \ldots, i_{r}$ satisfying $\sum_{s=1}^{r} i_{s}=1$. For any $\mathbf{m}=\left(m_{1}, \ldots, m_{r}\right) \in \mathbb{Z}^{r}$ satisfying $\sum_{s=1}^{r} m_{s}=0$, consider the $\mathbb{F}_{q}$-algebra homomorphism $\phi^{\mathbf{m}}: A \rightarrow K\{\tau\}$ given by

$$
\phi_{t}^{\mathbf{m}}=(-1)^{r-1} \prod_{s=1}^{r}\left(\tau-(-t)^{k_{s}}\right),
$$

where $k_{s}=i_{s}+m_{s}(q-1)$ for any $1 \leq s \leq r$. Now $\sum_{s=1}^{r} k_{s}=1$, so that the constant term of $\phi_{t}^{\mathbf{m}}$ is $(-1)^{r-1} \prod_{s=1}^{r}\left(-(-t)^{k_{s}}\right)=(-1)^{2 r} t=t$. Hence $\phi^{\mathbf{m}}$ is a rank-r Drinfeld module over $K$. 
Proposition 5.14. The isomorphism class $\left[\phi^{\mathbf{m}}\right]$ is contained in $\mathscr{D}(K, r, t)$. Moreover, the mod $t$ representation attached to $\phi^{\mathbf{m}}$ is of the form

$$
\bar{\rho}_{\phi^{\mathbf{m}}, t} \simeq\left(\begin{array}{cccc}
\chi_{t}^{i_{1}} & * & \cdots & * \\
& \chi_{t}^{i_{2}} & \ddots & \vdots \\
& & \ddots & * \\
& & & \chi_{t}^{i_{r}}
\end{array}\right),
$$

where $i_{1}, \ldots, i_{r}$ are the integers fixed as above.

Proof. For any finite place $v$ of $K$ not lying above $t$, since $-t \in \mathcal{O}_{K_{v}}$ and the leading coefficient of $\phi_{t}^{\mathbf{m}}$ is $(-1)^{r-1}$, we see that $\phi^{\mathbf{m}}$ has good reduction at $v$. Now $\phi^{\mathbf{m}}[t]$ coincides with the kernel of $\prod_{s=1}^{r}\left(\tau-(-t)^{k_{s}}\right)$. Applying Lemma 5.13 to $f_{s}=\left(\tau-(-t)^{k_{s}}\right) \cdots(\tau-$ $\left.(-t)^{k_{1}}\right)$, we see that $\bar{\rho}_{\phi^{\mathbf{m}}, t}$ is given as above since $\kappa_{\left((-t)^{\left.k_{s}\right)}\right.}=\chi_{t}^{k_{s}}=\chi_{t}^{i_{s}}$ for any $1 \leq s \leq r$.

PROPOSITION 5.15. If $r \geq 2$, then the set $\mathscr{D}(K, r, t)$ is infinite.

Proof. We construct an infinite subset of $\mathscr{D}(K, r, t)$ as follows. Fix $r$ integers $i_{1}, \ldots, i_{r}$ satisfying $\sum_{s=1}^{r} i_{s}=1$. For any positive integer $m$, consider $(-m, 0, \ldots, 0, m) \in \mathbb{Z}^{r}$ and define $\phi^{m}:=\phi^{(-m, 0, \ldots, 0, m)}$, which is a Drinfeld module satisfying $\left[\phi^{m}\right] \in \mathscr{D}(K, r, t)$ by Proposition 5.14. Write $\phi_{t}^{m}=t+c_{1} \tau+\cdots+c_{r-1} \tau^{r-1}+(-1)^{r-1} \tau^{r} \in K\{\tau\}$. Then by construction the coefficient $c_{r-1}$ is given by

$$
c_{r-1}=(-t)^{i_{1}-m(q-1)}+(-t)^{i_{r}+m(q-1)}+\sum_{s=2}^{r-1}(-t)^{i_{s}} .
$$

For any finite place $u$ of $K$ above $t$, if $m$ is sufficiently large, then

$$
u\left(c_{r-1}\right)=\left(i_{1}-m(q-1)\right) u(-t)<0 .
$$

Hence we see that $u\left(c_{r-1}\right) \rightarrow-\infty$ as $m \rightarrow \infty$. On the other hand, for two positive integers $m$ and $m^{\prime}$, if $\phi^{m^{\prime}}$ is isomorphic to $\phi^{m}$, then $\phi_{t}^{m^{\prime}}=x^{-1} \phi_{t}^{m} x$ for some $x \in \mathbb{F}_{K}^{\times}$by the same argument as in Example 5.11. These facts imply that, if $m^{\prime}$ is sufficiently large, then $\phi^{m}$ and $\phi^{m^{\prime}}$ are not isomorphic. Therefore the subset $\left\{\left[\phi^{m}\right] ; m \in \mathbb{Z}_{>0}\right\}$ of $\mathscr{D}(K, r, t)$ is infinite.

Acknowledgements. The author is grateful to his supervisor, Yuichiro Taguchi, for giving him useful advice about Drinfeld modules and for his guidance in preparing this paper. The author is greatly indebted to Akio Tamagawa for pointing out a mistake in an earlier version of this paper and for providing his idea to construct examples in Propositions 5.9 and 5.14. The author also would like to thank Yoshiyasu Ozeki for his helpful comments on Proposition 5.3. The author also thanks the referee, whose precise suggestions helped to improve the presentation of this paper.

\section{REFERENCES}

[An] G. W. Anderson. $t$-motives. Duke Math. J. 53 (1986), 457-502.

[Ar] K. Arai. Algebraic points on Shimura curves of $\Gamma_{0}(p)$-type. J. reine angew. Math. 690 (2014), 179-202. 
[Bo] A. Bourdon. A uniform version of a finiteness conjecture for CM elliptic curves. Math. Res. Lett. 22 (2015), 403-416.

[CL] I. Chen and Y. Lee. Explicit isogeny theorem for Drinfeld modules. Pacific J. Math. 263(1) (2013), 87-116.

[Dr] V. G. Drinfeld. Elliptic modules. Math. USSR Sb. 23 (1974), 561-592.

[Fa] G. Faltings. Finiteness theorems for abelian varieties over number fields. Arithmetic Geometry. Eds. G. Cornell and J. H. Silverman. Springer, Berlin, 1986, pp. 9-27.

[Go] D. Goss. Basic Structures of Function Field Arithmetic (Ergebnisse der Mathematik und ihrer Grenzgebiete, 35). Springer, Berlin, 1996.

[Ga1] F. Gardeyn. $t$-motives and Galois representations. PhD Thesis, Universiteit Gent, 2001.

[Ga2] F. Gardeyn. A Galois criterion for good reduction of $\tau$-sheaves. J. Number Theory 97 (2002), 447-471.

[Ga3] F. Gardeyn. The structure of analytic $\tau$-sheaves. J. Number Theory 100 (2003), 332-362.

[Ga4] F. Gardeyn. Analytic morphisms of $t$-motives. Math. Ann. 325 (2003), 795-828.

[Ih] Y. Ihara. Profinite braid groups, Galois representations and complex multiplications. Ann. of Math. (2) 123 (1986), 43-106.

[Ki] W. Kim. Galois deformation theory for norm fields and its arithmetic applications. Thesis, University of Michigan, 2009.

[KS] V. Kumer and J. Scherk. Effective versions of the Chebotarev density theorem for function fields. C. R. Acad. Paris Sér. I Math. 319(6) (1994), 523-528.

[Lo] D. Lombardo. On the uniform Rasmussen-Tamagawa conjecture in the CM case. Preprint. arXiv:1511.09019.

[Oz1] Y. Ozeki. Non-existence of certain Galois representations with a uniform tame inertia weight. Int. Math. Res. Not. 2011 (2011), 2377-2395.

[Oz2] Y. Ozeki. Non-existence of certain CM abelian varieties with prime power torsion. Tohoku Math. J. 65 (2013), 357-371.

[Ro] M. Rosen. Number Theory in Function Fields (Graduate Texts in Mathematics, 210). Springer, New York, 2002.

[RT1] C. Rasmussen and A. Tamagawa. A finiteness conjecture on abelian varieties with constrained prime power torsion. Math. Res. Lett. 15 (2008), 1223-1231.

[RT2] C. Rasmussen and A. Tamagawa. Arithmetic of abelian varieties with constrained torsion. Trans. Amer. Math. Soc. 369 (2017), 2395-2424.

[Se1] J.-P. Serre. Propriétés galoisiennes des points d'ordre fini des courbes elliptiques. Invent. Math. 15 (1972), 259-331.

[Se2] J.-P. Serre. Local Fields (Graduate Texts in Mathematics, 67). Springer, New York, 1979.

[ST] J.-P. Serre and J. Tate. Good reduction of abelian varieties. Ann. of Math. (2) 88 (1968), 492-517.

[Ta] T. Takahashi. Good reduction of elliptic modules. J. Math. Soc. Japan 34(3) (1982), 475-487.

\author{
Yoshiaki Okumura \\ Department of Mathematics \\ Tokyo Institute of Technology \\ 2-12-1 Oh-okayama, Meguro-ku \\ Tokyo 152-8551 \\ Japan
}

(E-mail address: okumura.y.ab@m.titech.ac.jp) 